\title{
A quantitative analysis of environmental associations in sauropod dinosaurs
}

\author{
Philip D. Mannion and Paul Upchurch
}

\begin{abstract}
Both the body fossils and trackways of sauropod dinosaurs indicate that they inhabited a range of inland and coastal environments during their 160-Myr evolutionary history. Quantitative paleoecological analyses of a large data set of sauropod occurrences reveal a statistically significant positive association between non-titanosaurs and coastal environments, and between titanosaurs and inland environments. Similarly, "narrow-gauge" trackways are positively associated with coastal environments and "wide-gauge" trackways are associated with inland environments. The statistical support for these associations suggests that this is a genuine ecological signal: non-titanosaur sauropods preferred coastal environments such as carbonate platforms, whereas titanosaurs preferred inland environments such as fluvio-lacustrine systems. These results remain robust when the data set is time sliced and jackknifed in various ways. When the analyses are repeated using the more inclusive groupings of titanosauriforms and Macronaria, the signal is weakened or lost. These results reinforce the hypothesis that "wide-gauge" trackways were produced by titanosaurs. It is commonly assumed that the trackway and body fossil records will give different results, with the former providing a more reliable guide to the habitats occupied by extinct organisms because footprints are produced during life, whereas carcasses can be transported to different environments prior to burial. However, this view is challenged by our observation that separate body fossil and trackway data sets independently support the same conclusions regarding environmental preferences in sauropod dinosaurs. Similarly, analyzing localities and individuals independently results in the same environmental associations. We demonstrate that conclusions about environmental patterns among fossil taxa can be highly sensitive to an investigator's choices regarding analytical protocols. In particular, decisions regarding the taxonomic groupings used for comparison, the time range represented by the data set, and the criteria used to identify the number of localities can all have a marked effect on conclusions regarding the existence and nature of putative environmental associations. We recommend that large data sets be explored for such associations at a variety of different taxonomic and temporal scales.
\end{abstract}

Philip D. Mannion and Paul Upchurch. Department of Earth Sciences, University College London, Gower Street, London WC1E 6BT, United Kingdom.E-mail:p.mannion@ucl.ac.uk; p.upchurch@ucl.ac.uk

Accepted: 9 September 2009

\section{Introduction}

Sauropods were a major Mesozoic radiation of gigantic herbivorous dinosaurs and included the largest known terrestrial animals (Wilson 2002; Upchurch et al. 2004) (Fig. 1). They make their first appearance in the fossil record in the Late Triassic (Buffetaut 2000; Yates and Kitching 2003), as also inferred from ghost ranges (Upchurch 1995; Sereno 1999), and had achieved a global distribution (excluding Antarctica) by the Middle Jurassic (McIntosh 1990; Upchurch et al. 2002; Weishampel et al. 2004a). A major extinction event occurred at the Jurassic/Cretaceous boundary, with $60 \%$ of lineages and $80 \%$ of genera disappearing at this time (Upchurch and Barrett 2005; Barrett et al. 2009). During the Early Cretaceous, the remaining sauropod lineages gradually dwindled to extinction, except for the rebbachisaurids and titanosaurs (Fig. 2). Members of the latter group were extremely rare in the Jurassic, but this clade diversified during the Cretaceous, producing over 50 genera (Salgado et al. 1997; Wilson and Upchurch 2003; Upchurch et al. 2004; Curry Rogers 2005). Titanosaurs were a significant part of many terrestrial ecosystems before becoming extinct at the end of the Cretaceous along with the other non-avian dinosaurs (Salgado 2001).

Over the past two decades, our knowledge of sauropod paleoecology has been improved by the discovery of new material, the development of taxonomic schemes based on detailed phylogenetic analyses, and investigations of the depositional environments that 


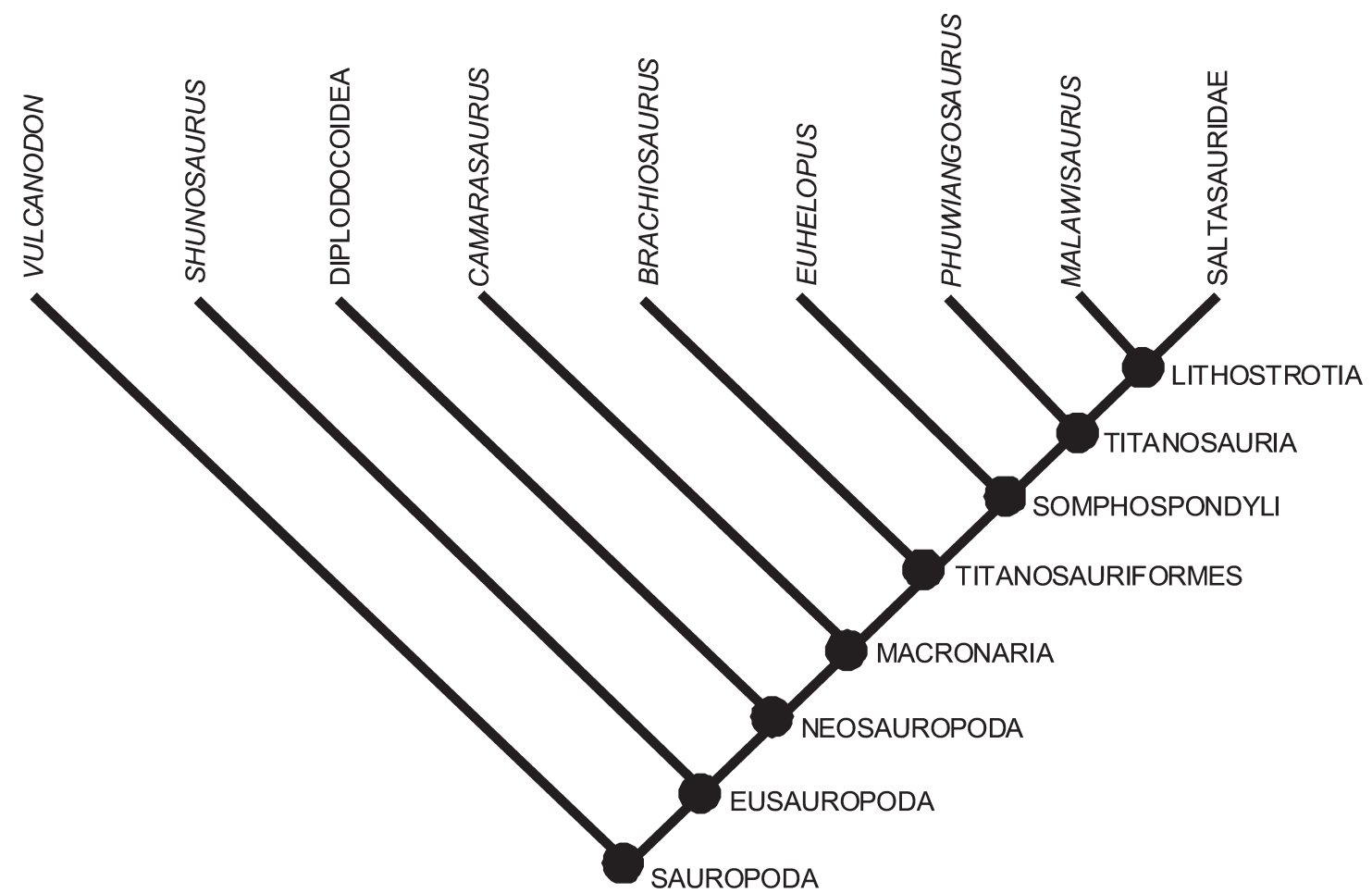

FIGURE 1. A simplified cladogram showing the relationships between the major sauropod lineages and the stem- and node-based names currently in use (based on Wilson and Upchurch 2003, 2009, and Upchurch et al. 2004).

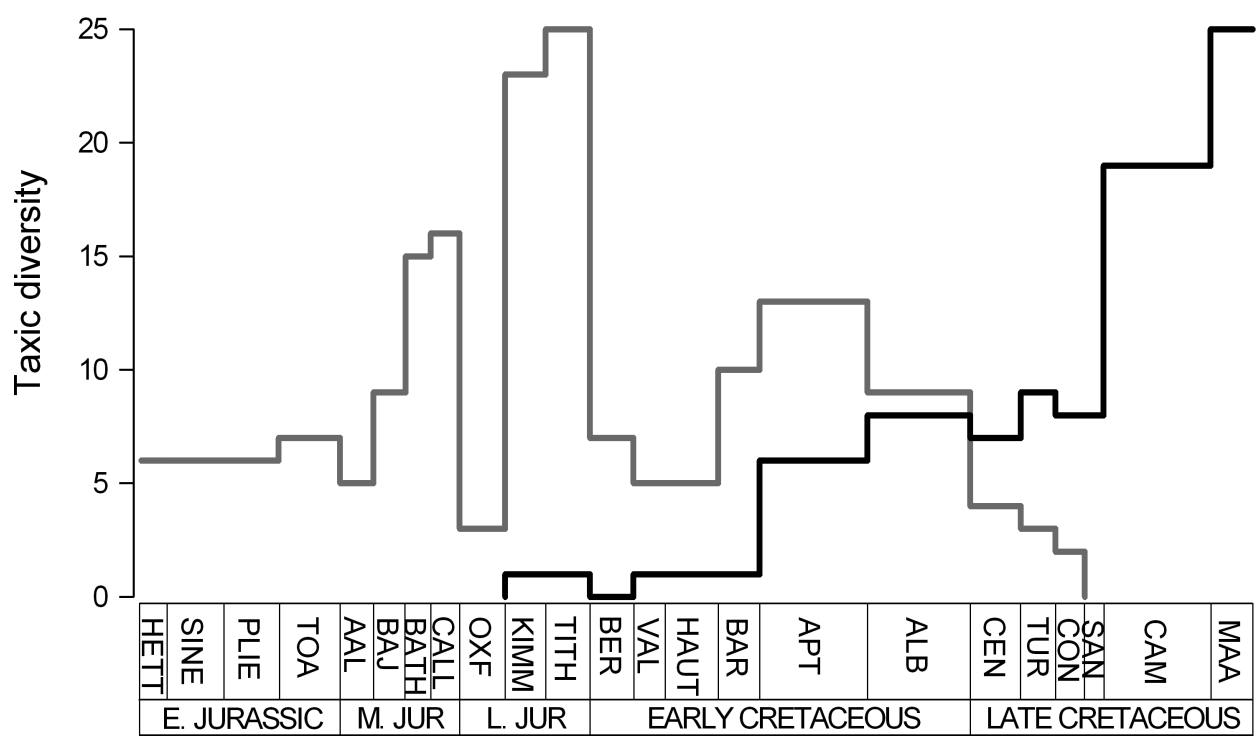

FIGURE 2. Taxic diversity curves for non-titanosaur (gray line) and titanosaur (black line) genera during the Jurassic and Cretaceous (based on the information on generic occurrences in the data set utilized in the current study-see Supplementary data). Data are plotted against the absolute timescale of Gradstein et al. (2004). Abbreviations: Hett, Hettangian; Sine, Sinemurian; Plie, Pliensbachian; Toa, Toarcian; Aal, Aalenian; Baj, Bajocian; Bath, Bathonian; Call, Callovian; Oxf, Oxfordian; Kimm, Kimmeridgian; Tith, Tithonian; Ber, Berriasian; Val, Valanginian; Haut, Hauterivian; Bar, Barremian; Apt, Aptian; Alb, Albian; Cen, Cenomanian; Tur, Turonian; Con, Coniacian; San, Santonian; Cam, Campanian; Maa, Maastrichtian. 
yield sauropod body fossils and trackways (see reviews in Upchurch et al. 2004; Carpenter and Tidwell 2005; Curry Rogers and Wilson 2005). Paleoenvironmental analyses demonstrate that sauropods inhabited a wide variety of environments ranging from floodplain and fluvio-lacustrine settings in the Morrison Formation of North America, to nearshore estuarine conditions in the Tendaguru Formation of Tanzania (Dodson et al. 1980; Russell et al. 1980). However, it is difficult to generalize about sauropods as a whole, or identify the habitat preferences of groups within Sauropoda, because most paleoecological studies have focused on a single geological formation (e.g., Dodson et al. 1980) or geographical area (e.g., Lehman 1987; Lucas and Hunt 1989) (see also Butler and Barrett 2008 for a discussion of this issue). Some of the key questions that remain to be answered include:

- What, if any, habitat preferences existed among different sauropod groups?

- Can we detect evidence for true habitat preferences against the background "noise" generated by our patchy and biased sampling of the fossil record?

- How did habitat preferences relate to the morphological adaptations displayed by each type of sauropod?

- Did habitat preferences change during sauropod evolution?

- Did habitat preferences constrain and/or promote changes in sauropod diversity and geographic distribution?

Only a few studies have attempted to address these questions through quantitative analyses using extensive data sets. For example, Lockley et al. (1994) used the trackway record to demonstrate that (1) sauropods often walked on submerged substrates in coastal and deltaic settings; (2) tracksites are mainly associated with tropical and subtropical latitudes (mean average Northern Hemisphere paleolatitude $=25^{\circ}$ ); and (3) the majority of trackways occur in semiarid or seasonal climatic environments where carbonate deposition was taking place (i.e., in lacustrine settings or in marine carbonate platform environments). Butler et al. (2007) and Butler and Barrett (2008), using an extensive data set of Cretaceous dinosaurs, confirmed the strength of the sauropod ichnofossil record in coastal environments. However, they disagreed that sauropod trackways were overrepresented in coastal environments relative to trackways of other herbivorous groups and suggested that the preservation potential for footprints was enhanced in these habitats. The body fossil evidence, they maintained, suggested "more distal, or inland (away from channels), paleoenvironmental preferences for sauropods, at least during the Cretaceous, when compared to contemporaneous clades such as Nodosauridae and Hadrosauridae" (Butler and Barrett 2008: pp. 1030-1031). Thus, both Lockley et al. (1994) and Butler and Barrett (2008) focused on the environments occupied by sauropods as a whole, and discussion of "within sauropod" patterns was limited to the distributions of Macronaria versus nonMacronaria (see Fig. 1).

Our study uses a large data set comprising the depositional environments for virtually all identifiable sauropod trackways and body fossils. We compare the distributions of "narrow-gauge" trackways and non-titanosaur body fossils with those of "wide-gauge" trackways and titanosaur body fossils, using Pearson's chi-square tests to evaluate the statistical support for putative nonrandom associations. Finally, we evaluate the implications of our results for understanding the habitat preferences of sauropods, and discuss their relevance to existing hypotheses of sauropod paleoecology and evolutionary history.

\section{Materials and Methods}

Data

Exploring the possible environmental preferences in extinct terrestrial vertebrates requires information on the spatiotemporal distributions of fossils and their depositional environments. We have constructed a global database of all sauropod occurrences, including detailed information on geographic location, geological setting, paleoenvironmental indicators, stratigraphic age, and taxonomic 

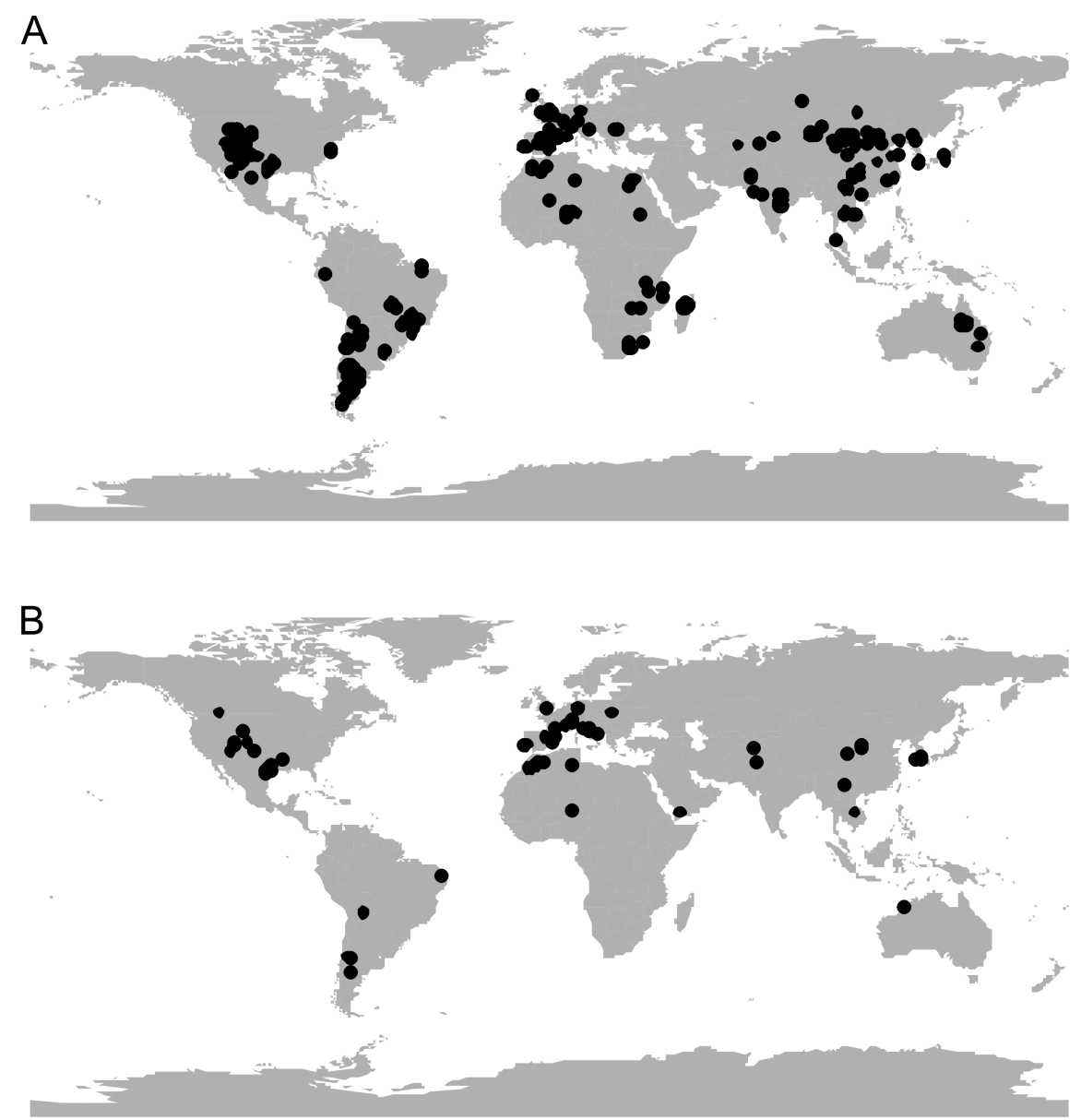

FIGURE 3. Distribution of all sauropod occurrences used in this study, plotted on modern-day world maps. A, Distribution of sauropod body fossil localities. B, Distribution of sauropod tracksite localities. Images produced in ArcGIS.

composition. These data were gathered from the primary literature, the Paleobiology Database (www.paleodb.org; Carrano 2008), and personal observations of specimens in museum collections. This global data set was then filtered to remove tracksites and body fossils of dubious sauropod affinity and those occurrences identified only as "Sauropoda indet." The resulting database (see Supplementary Materials online at http://dx.doi. org/10.1666/08085.s1) contains information from 896 localities (706 body fossil localities and 190 tracksites; Fig. 3) that is estimated to represent approximately 1988 sauropod individuals (1355 based on body fossils and 633 trackmakers). The criteria for recognizing separate localities and individuals are discussed in "Units of Analysis" below.
Methodological Approach and Analyses

Analytical Protocol and Statistical Evaluation.-The rationale for investigating and testing environmental associations is as follows. Suppose we have two environments (A and $B$ ) and two types of organism ( $X$ and $Y$ ). The null hypothesis is that there is no detectable environmental preference or association: that is, $X$ and $Y$ are distributed across $A$ and $B$ with no significant skew or bias. In ecological studies, this null hypothesis can be tested using a variety of statistical tests, the two most commonly used being the G-test and Pearson's chi-square test (Sokal and Rohlf 1987; Waite 2000; Hammer and Harper 2006). Although the two tests, which are both used to determine the "goodness of fit" between observed and expected values, 
generally give the same results (Sokal and Rohlf 1987), the G-test has the disadvantage of being impossible to calculate if some observed values are zero, because logarithms are used in its calculation (Waite 2000). Because many of our analyses include observed values of zero, Pearson's chi-square test is the more appropriate method for this study. The use of chi-square tests in paleoenvironmental analyses also has precedents in fossil invertebrate studies (e.g., Peters and Bork 1999; Scholz and Hartman 2007; De Francesco and Hassan 2008) as well as the dinosaur analysis of Butler and Barrett (2008). In the context of paleoecology, uneven sampling of fossils from different environments introduces a complicating factor. If we have collected three times as many fossils of group $X$ from environment $A$ relative to environment $B$, then a non-skewed distribution would be one in which three times as many members of group $X$ are found in $A$ compared to $B$. We must therefore take into account the relative sampling of our taxon types and environmental categories when calculating the expected number of occurrences. Thus, the expected number of occurrences of taxon $X$ in environment $A\left(E_{X A}\right)$ is given by

$$
E_{X A}=N_{T} \times\left[\left(N_{X} / N_{T}\right) \times\left(N_{A} / N_{T}\right)\right],
$$

where $N_{X}$ is the number of occurrences of members of group $X, N_{A}$ is the number of occurrences of environment $A$, and $N_{T}$ is the total number of occurrences (modified from Waite 2000: see also Butler and Barrett 2008). Equation (1) simplifies to

$$
E_{X A}=\left(N_{X} \times N_{A}\right) / N_{T} .
$$

The other three expected values required for the chi-square test are given by

$$
\begin{aligned}
& E_{X B}=\left(N_{X} \times N_{B}\right) / N_{T} \\
& E_{Y A}=\left(N_{Y} \times N_{A}\right) / N_{T} \\
& E_{Y B}=\left(N_{Y} \times N_{B}\right) / N_{T} .
\end{aligned}
$$

The formulae above were used to calculate the expected values shown in Tables 2-10. For example, if we designate $X$ and $Y$ to represent narrow- and wide-gauge trackways respectively, and $A$ and $B$ to represent inland and coastal localities where such tracks are found, then $E_{X A}$ is the expected number of times that narrow-gauge trackways should occur in inland environments if there is no skew in the distribution. Thus, using the observed values listed for Analysis 5 (Table 2$), E_{X A}=(41 \times 116) / 190=25.032$ (compared to the observed value of 14).

In this study, we have applied the chisquare test 134 times in order to explore different aspects of our data set (see below). Therefore, if we used the standard $p$-value for statistical significance of 0.05 we risk incorrectly attributing significance to some of our pairwise comparisons. Two tests are commonly used for identifying which of these pairs of samples are significantly different. The first of these, Tukey's HSD, has the disadvantage of reporting too high $p$-values when sample sizes are unequal (Hammer and Harper 2006). The Bonferroni test also subjects the samples to pairwise comparisons, akin to the chi-square analyses, but uses much lower significance levels (Rice 1989) and is thus the more appropriate method here. This correction states that the $p$-value for determining statistical significance is given by $\alpha / n$, where $\alpha$ is the original desired $p$-value (in this case 0.05 ) and $n$ is the number of analyses $(=134)$. Thus, the $p$-value we have used to determine statistical significance is $3.73 \times 10^{-4}$. By lowering the significance level, the Bonferroni correction greatly reduces the likelihood of incorrectly attributing significance to our pairwise comparisons (Waite 2000). A second advantage of the Bonferroni correction is that it can be used when the multiple analyses are independent or non-independent from each other, as is the case in this study (see "Analyses and Results" below).

Units of Analysis.-In order to study environmental associations using chi-square analyses we require counts of the number of times a taxon occurs in a particular environment, which can be estimates of either (1) the number of individual organisms belonging to a given taxon present in each habitat type, or (2) the number of localities where a given taxon occurs in each habitat type. Each type of 
estimate has its advantages and disadvantages.

Accurate estimation of the number of individual fossil organisms is often problematic, especially when dealing with fragmentary vertebrate and plant remains. In this study we estimated the minimum number of individuals (MNIs) for each locality. The use of MNIs is a standard tool in archaeological, paleoecological and taphonomic studies (e.g., Grayson 1973; Badgley 1986; Gilinsky and Bennington 1994; Davis and Pyenson 2007). For example, if a quarry consisted of closely associated material belonging to the same taxon, with no duplication or size variation of elements, then we would consider this a single individual. However, this method of estimating the MNIs does have the disadvantage of unavoidably undercounting the number of individuals. Estimating the MNIs from trackways can be even more difficult because a single trackmaker could have made many different tracks, and the relative size of foot impressions can be affected by under-printing and over-printing (Day et al. 2002, 2004; Milàn and Bromley 2006). Without information on the tracksite that indicated otherwise, we assumed that a tracksite represented no more than one trackmaker per recognizable taxon. This assumption was made even when an author stated that a locality had produced "trackways" (e.g., Lockley et al. 2006). Future analyses might wish to assume the presence of two individual trackmakers in such situations, though we doubt that this refinement would have a major effect on our conclusions because it would add roughly equal numbers of narrow- and wide-gauge individual trackmakers to both coastal and inland environments.

Although locality-based estimates avoid such problems of estimating number of individuals (particularly for trackway data), boundaries between "separate" localities can be somewhat arbitrary. For example, areas such as Como Bluff in the Late Jurassic Morrison Formation of North America have produced sauropod material from approximately the same horizons in several closely situated quarries (Ostrom and McIntosh 1966). In our study, localities are based on the divisions used in recent data sets (e.g., Lockley et al. 1994; Weishampel et al. 2004a; Carrano 2008; www.paleodb.org) and are defined as separate geographic locations and discrete stratigraphic levels that yield body fossils and/or trackways (modified from Lockley et al. 1994: p. 234). Each locality is counted only once, irrespective of the number of individuals present.

Environmental Categories.-The depositional environments that have yielded body fossils and trackways have been allocated to one of two broad categories: "inland" and "coastal." Sauropod fossils are occasionally recovered from marine deposits ( $0.01 \%$ of occurrences), but we excluded these because it seems highly unlikely that sauropods could have lived in marine environments. Inland environments include fluvial, lacustrine, floodplain and eolian settings; coastal environments comprise estuarine, deltaic, lagoonal, and carbonate platform settings. The division into inland versus coastal is only one of the many possible ways of combining the different environments into categories for the purposes of analysis. We focus on the inland/coastal division because these categories have yielded environmental associations among other dinosaurs (Butler et al. 2007; Butler and Barrett 2008) and because previous studies of sauropod paleoecology have made claims concerning preferences for one of these broad habitat types (e.g., Lehman 1987; Lucas and Hunt 1989).

Taxonomic Categories.-The search for environmental associations within Sauropoda requires that the taxa and trackways be divided into at least two distinct types or categories. At present, most sauropod trackways can only be placed in one of two (or perhaps three) broad categories: i.e., narrowgauge and wide-gauge with, or without, manus-claw and phalangeal impressions (Fig. 4) (Farlow et al. 1989; Lockley et al. 1994; Wilson and Carrano 1999; Day et al. 2004). Narrow-gauge trackways have footprints that approach (or intersect) the midline, with pollex claw impressions preserved (e.g., Parabrontopodus: Fig. 4A), whereas widegauge trackways have footprints placed well away from the midline and pollex claw 
A

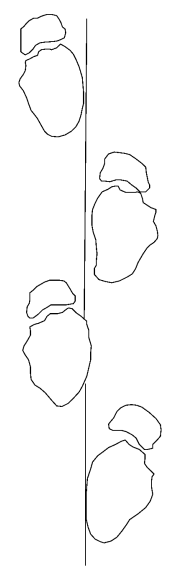

\section{C}

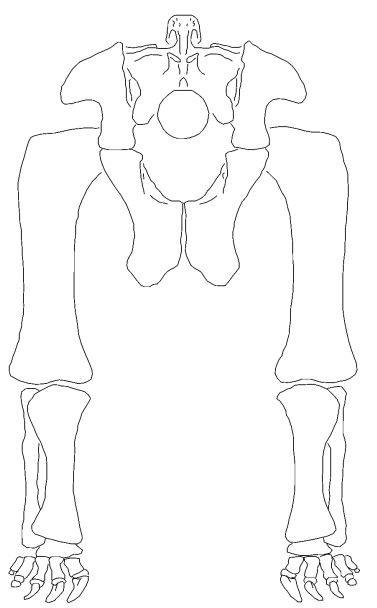

B

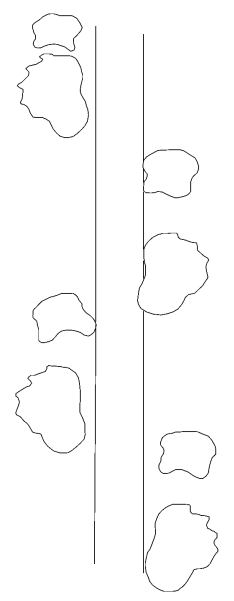

D

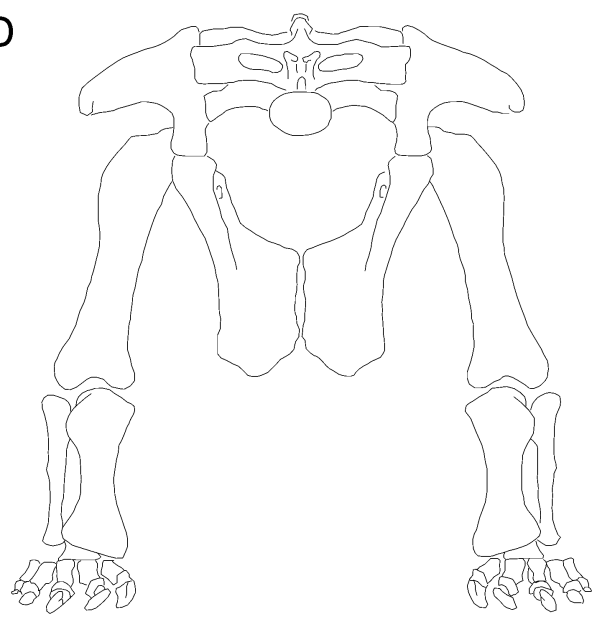

FigurE 4. The two main sauropod track types: "narrow-gauge" trackway (A) and "wide-gauge" trackway (B). Reconstructed pelvic girdles and hindlimbs, in anterior view, of the non-titanosaur Camarasaurus (C) and the titanosaur Opisthocoelicaudia (D). The vertical lines in A and B illustrate the distance separating left and right prints in the trackways. Images modified from Lockley et al. (1994) and Wilson and Carrano (1999).

impressions are frequently absent (e.g., Brontopodus: Fig. 4B). On the basis of several femoral morphological features (such as the lateral deflection of the proximal one-third of the femur) which suggest that titanosaurs held their limbs farther from the midline than other sauropods (Fig. 4C,D), Wilson and Carrano (1999) argued that titanosaurs were responsible for producing these wide-gauge tracks. This interpretation is also supported by the derived loss of pollex claws and manual phalanges in both titanosaurian skeletal remains and many wide-gauge trackways (Salgado et al. 1997; Day et al. 2002), though this may not be the case in basal titanosaurs or the more inclusive Titanosauriformes
(Fig. 1) (Day et al. 2004). It is difficult to assign sauropod trackways to their trackmaker more precisely than either narrowgauge or wide-gauge types because of the very limited number of derived features in the fore and hind feet of distinct sauropod lineages that can be unambiguously recognized in trackways (though see Wilson 2005a; Wright 2005). However, when trace fossils can be accurately assigned to particular taxa, analyses of separate body fossil and trace fossil data sets have the advantage that they yield two independent assessments of the environmental associations for the organisms concerned. Such an approach might reinforce the support for the hypothesis that a given 
clade made the trace fossil, or might reveal incongruence that would require reassessment of the initial identification of the putative trace fossil makers. If congruence between the body fossil and trace fossil signals exists, it is then justifiable to unite the data sets to produce a "combined evidence" analysis of the environmental associations. Thus, trackways and body fossils have their own particular advantages and disadvantages for paleoecological research and we therefore analyze both types of data separately and in combination.

With regard to body fossils, there is a much wider choice of possible comparisons, such as neosauropods versus non-neosauropods, titanosaurians versus non-titanosaurians, or diplodocoids versus macronarians. In this study, the majority of the analyses examine the environmental associations of titanosaurs versus non-titanosaurs. This enables us to carry out "combined evidence" analyses in which narrow-gauge trackways and nontitanosaur body fossil data are combined and compared with wide-gauge trackways plus titanosaur body fossil data, providing two independent lines of evidence to test for environmental associations. In addition, the utilization of narrow- and wide-gauge locomotor styles might be related to the biomechanical demands of different habitats, making it a suitable starting point for investigating environmental associations among sauropods.

Wilson and Carrano (1999) noted that basal titanosauriforms such as Brachiosaurus and Euhelopus have femoral morphologies that are intermediate between those in narrow-gauge non-titanosaurs and wide-gauge titanosaurs. We therefore also analyze the body fossil data using a division into non-titanosauriforms and titanosauriforms, as well as a partition into non-titanosaurian titanosauriforms and titanosaurs to test for differences within Titanosauriformes.

Macronaria is a major sauropod clade that is largely composed of the Titanosauriformes and a few basal genera such as Camarasaurus (Fig. 1). A recent study of Cretaceous dinosaurian distributions suggested that "Sauropods show little evidence for broad environmental associations: a significant negative association between Macronaria and coastal environments may be a result of taphonomic processes" (Butler et al. 2007: pp. 54-55). Furthermore, Butler and Barrett (2008: Table 1) also reported a statistically significant $\left(p<1 \times 10^{-4}\right)$ positive association between Macronaria and inland environments. We test this possibility by comparing macronarians with non-macronarians and by restricting our data set to Cretaceous occurrences. In addition, we reanalyze the Butler and Barrett (2008) data set to look for patterns at the taxonomic levels of titanosauriforms and titanosaurs.

A Note on Paraphyly.-Division of the sauropod data into non-titanosaurs versus titanosaurs, non-titanosauriforms versus titanosauriforms and macronarians versus nonmacronarians means that we are comparing the distributions of a paraphyletic assemblage with those of a monophyletic group. The use of paraphyletic groups in paleobiological analyses is potentially problematic because such groups have an arbitrary taxonomic content. If we choose to use a different taxonomic definition, the boundaries around the paraphyletic assemblage change and therefore its contents also change. However, the use of paraphyletic groups is justified when such assemblages represent evolutionary grades or ecological communities (see Peters [2008] for an example concerning Sepkoski's [1984] marine invertebrate evolutionary faunas, and Wilson and Carrano [1999] who carried out statistical analyses of femoral measurements based on a division into "titanosaurs" and "other sauropods"). For example, when analyzing the ecological consequences of limblessness, it would be legitimate to compare one or more of the monophyletic limbless squamate groups (such as snakes, amphisbaenians) with the paraphyletic grade of "lizards" in which limbs are retained. In such analyses, the boundaries of a paraphyletic group are not arbitrary because they are set by the retention of one or more symplesiomorphies that are related to the biomechanics and/or ecology of the group. The synapomorphies which mark the boundary between a paraphyletic grade and one of its monophyletic descendant clades might relate to an 
evolutionary shift in physiology, behavior, habitat, etc., so that the only meaningful way to explore the resulting differences in environmental association would be to compare the distributions of the paraphyletic assemblage and the monophyletic group. It is conceivable that the acquisition of the wide-gauge stance in titanosaurs enabled them to invade or inhabit a different set of environments, and we therefore believe that our comparisons among sauropods are potentially ecologically meaningful.

Uneven Sampling of Environments through Time.-Trends in the diversity and abundance of taxa through time, combined with uneven sampling of depositional environments, could create an additional problem for paleobiologists searching for environmental associations. Suppose, for example, that the ratio of inland to coastal localities sampled from the Jurassic is 1:4 and from the Cretaceous is $4: 1$. Furthermore, suppose that titanosaurs were rare during the Jurassic and common during the Cretaceous relative to non-titanosaurs, so that the ratio of titanosaurs to non-titanosaur individuals is $1: 10$ in the Jurassic and 10:1 in the Cretaceous. Finally, suppose that titanosaurs and nontitanosaurs exhibited no environmental preferences so that they were evenly distributed across the two environmental categories. Under this scenario, if we collected 110 sauropod individuals from the Jurassic, approximately ten of these would be titanosaurs and 100 would be non-titanosaurs. If these taxa display no environmental preferences, then we would expect eight titanosaurs and 80 non-titanosaurs from the coastal localities and two titanosaurs and 20 non-titanosaurs from the inland localities. If we then collected 110 sauropod individuals from the Cretaceous, we would expect 80 titanosaurs and eight non-titanosaurs from the inland localities, and 20 titanosaurs and two non-titanosaurs from the coastal localities. The total (Jurassic + Cretaceous) data set would comprise 82 titanosaurs and 28 non-titanosaurs from inland localities, and 28 titanosaurs and 82 non-titanosaurs from coastal localities. This skewed distribution passes the chi-square test $\left(p<1 \times 10^{-5}\right)$, but the uneven distribution does not result from environmental preferences: such a skew is a by-product of the combination of long-term trends in the relative abundances of the two taxon categories and two environmental categories. Thus, given admittedly somewhat contrived conditions, a statistically robust, but nonetheless artifactual, environmental association can be generated.

Evidence suggests that investigations of environmental associations in sauropods must deal with precisely the scenario outlined above. Our current understanding of sauropod diversity patterns is that non-titanosaur lineages were diverse during the Jurassic, declined in the Early Cretaceous, and became extinct in the early Late Cretaceous, whereas titanosaurs were rare in the Jurassic but increased in diversity and abundance in the Cretaceous (Upchurch and Barrett 2005) (Fig. 2). To investigate the possible effects of fluctuations of inland and coastal environments, we calculated the relative sampling rates of these two environments for sauropodbearing localities for each stage of the Jurassic and Cretaceous. However, to avoid the potential circular reasoning that arises from only considering sauropod-bearing localities (i.e., it is possible that there were as many coastal localities capable of preserving largebodied terrestrial vertebrates during the Cretaceous as there were during the Jurassic, but we might recognize fewer of them during the Cretaceous because we have collected data only on sauropod-bearing localities and because titanosaurs preferred inland habitats), we have also compiled information on the numbers of ornithischian-bearing localities (www.pbdb.org; Carrano 2008).

There is a marked difference in the relative sampling rates for coastal and inland sauropod-bearing localities during the Jurassic and Cretaceous. Table 1 shows that the percentage of coastal sauropod-bearing localities during the Jurassic is $19.4 \%$, whereas during the Cretaceous this falls to $8.4 \%$. For numbers of ornithischian-bearing localities, Table 1 shows that coastal environments contribute $23.2 \%$ of Jurassic and only $7.5 \%$ of Cretaceous localities. Given that several ornithischian clades apparently display a 
TABLE 1. The relative abundances of inland and coastal environments during the Late Triassic to Late Cretaceous, based on the numbers of localities producing sauropod body fossils, ornithischian body fossils, and combined sauropod and ornithischian body fossils.

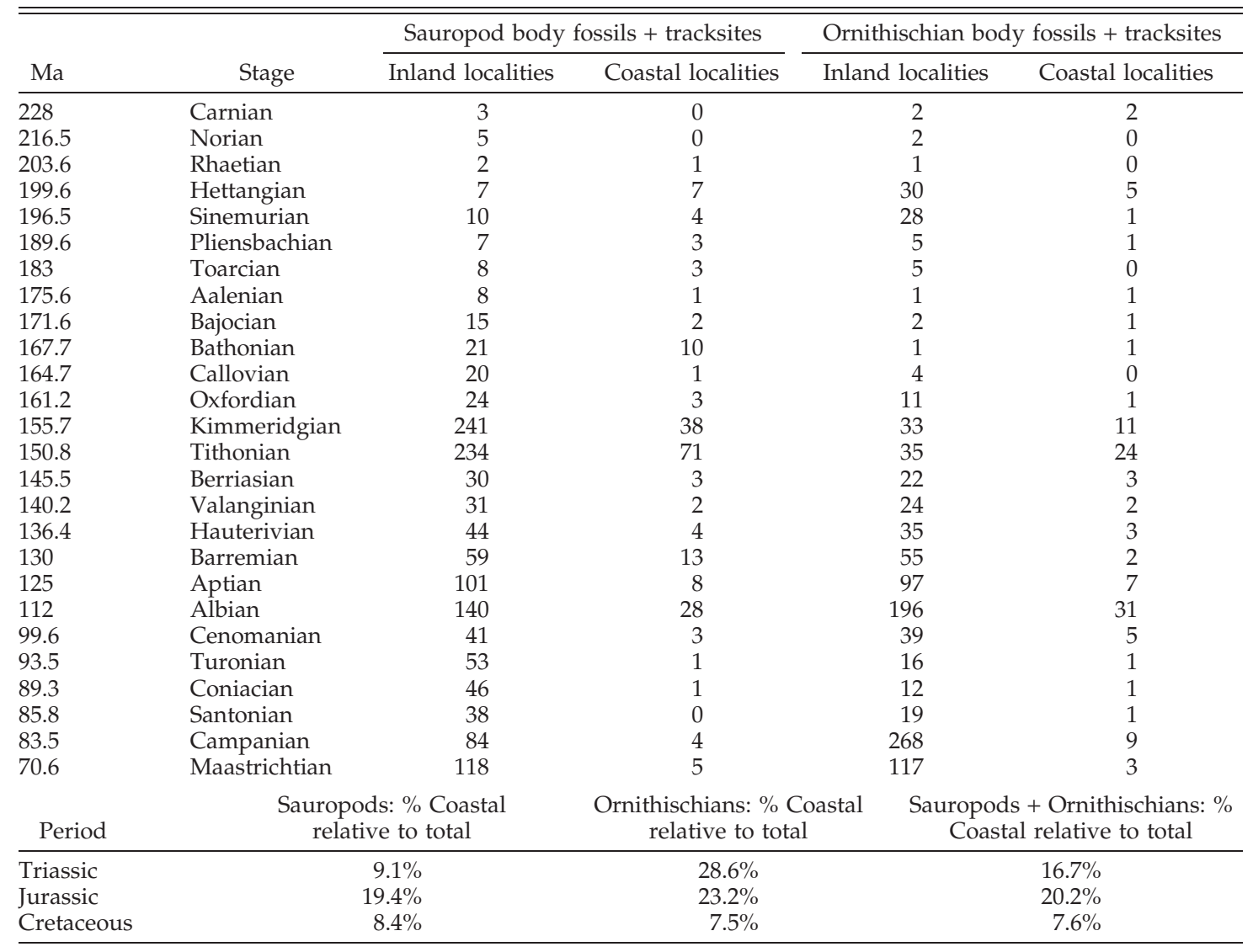

positive association with marine/coastal localities during the Cretaceous (Butler and Barrett 2008), the sauropod and ornithischian data taken together and separately (Table 1) suggest that coastal localities are indeed underrepresented during the Cretaceous relative to the Jurassic. Thus, we need to eliminate the possibility that statistically significant environmental associations relating to titanosaurs versus non-titanosaurs are an artifact of uneven sampling of inland and coastal habitats during the Jurassic and Cretaceous.

The deleterious effects of trends in the relative abundance and/or diversity of taxa and environments can be ameliorated by searching for environmental associations using narrower time bins. If titanosaurs and non-titanosaurs displayed genuine habitat preferences, then statistically robust environ- mental associations should persist even when the data are drawn from a narrower time window in which the relative abundance of inland and coastal environment types does not change markedly. We therefore repeat the chi-square analyses using Cretaceous, Earlyearly Late Cretaceous (Berriasian-Coniacian) and stage-level time slices.

"Jackknifing" the Data.-One danger with our approach is that the results may be dominated by short-lived windows of exceptional preservation that overwhelm signals in the rest of the data set. This is a particular concern with our trackway data because 73 out of 190 localities (38.4\%) are based on a mega-tracksite from the Early Cretaceous of the Republic of Korea (Lim et al. 1989; Lee et al. 2000; Lockley et al. 2006). Thus, the decision to treat this mega-tracksite as 73 separate localities, rather than one large 
locality, could make an important difference to our results (see "Units of Analysis" above). We have therefore restricted analysis of trackway data to the 118 localities that remain after removal of all but one of the Korean tracksites. However, subsuming 73 separate Korean tracksites into just one locality could be regarded as overly severe, given that at least six main tracksites can be distinguished from each other on the basis of spatial and stratigraphic separation (Lim et al. 1989; Lee et al. 2000; Lockley et al. 2006). Therefore, we have also run the analyses with Korean tracksites represented by these six regions (see also Wright 2005). Finally, the U.S. track record also constitutes a large proportion (17\%) of the tracksite data set, including a large number of Early Cretaceous coastal localities; thus, our analyses have been run with and without these tracksites.

\section{Analyses and Results}

The 134 analyses outlined below have been divided into three groups. Those in the first group (nos. 1-14, Table 2) use the whole Mesozoic data set to determine whether titanosaurs (or one of the slightly more inclusive groups represented by the Titanosauriformes and Macronaria) and wide-gauge trackways occur more often than expected in either inland or coastal environments. Because these analyses are based on the most data, we give them the greatest weight in our discussion of the implications of our results. Analyses in the second group (nos. 15-115, Tables 3-9) examine the effects of time-slicing the data sets. Those in the final group (Analyses 116-134, Table 10) determine the effects of jackknifing the trackway data by removing the U.S. and South Korean localities. It should be noted that the 134 separate analyses have complex relationships in terms of their independence or non-independence. Many of the time slice analyses are effectively independent of each other; for example, Bathonian and Callovian stage data sets (e.g., nos. 45 and 46) share no data points in common. Similarly, analyses based solely on body fossil data (e.g., nos. 1 and 4) are independent of those based purely on track- way data (e.g., nos. 2 and 5). However, many other analyses are wholly or partly nonindependent; for example, the data in Analysis 4 (all body fossil localities; Table 2) overlap substantially with those in Analysis 18 (Cretaceous body fossil localities; Table 3). The number of data supporting each statistically significant result, and the independence of separate analyses, will be considered when we discuss the implications of the results.

\section{Analyses 1-14: Mesozoic Data Sets}

Titanosaurs versus Non-Titanosaurs.-Analyses 1-6 (Table 2) compare the distributions of titanosaur and non-titanosaur body fossils, narrow-gauge and wide-gauge trackways, and body fossils plus trackways, based on estimated numbers of individuals (Analyses 1-3) and localities (Analyses 4-6). Analyses 15 produce very low $p$-values $\left(p=1 \times 10^{-5}\right.$ to 7 $\left.\times 10^{-4}\right)$, whereas Analysis 6 is non-significant $(p<0.0677)$. These results suggest that titanosaurs and wide-gauge tracks occur more often than expected in inland environments, whereas non-titanosaurs and narrowgauge trackways occur more often than expected in coastal environments.

Titanosauriforms versus Non-Titanosauriforms.-Analyses 7-10 (Table 2) examine possible environmental associations among titanosauriform and non-titanosauriform body fossil data by themselves and with the trackway data added, based on estimated numbers of individuals and localities. Three of these analyses (7, 9 and 10) fail to produce statistically significant results ( $p=0.1296$ to 0.2537$)$. However, results of Analysis 8, which combines body fossil and trackway data for individuals, are significant $(p$ $=2 \times 10^{-4}$ ), suggesting that titanosauriforms occur more often than expected in coastal environments, whereas non-titanosauriforms occur more often than expected in inland environments. This is the reverse of the environmental association found among titanosaurs and non-titanosaurs, which we hypothesize occurs because the data from non-titanosaurian ("basal") titanosauriforms is somehow "swamping" the inland signal recovered in Analyses 1-5 (see below).

To test for this swamping by non-titanosaurian titanosauriforms, we restricted the 
TABLE 2. Summary of analyses (1-14) of potential environmental associations among sauropods, based on the complete Mesozoic data sets (see "Analyses and Results" for details). Abbreviations: E, expected number; O, observed number; $\mathrm{T}$, total number. The following symbols next to an analysis number denote: (1) an asterisk $\left({ }^{*}\right)$ denotes a significant result which supports titanosaurs preferring inland environments; (2) two asterisks (**) denote a significant result which supports titanosaurs preferring coastal environments; (3) a hash sign (\#) denotes a significant result which supports titanosauriforms preferring inland environments; (4) two hash signs (\#\#) denote a significant result which supports titanosauriforms preferring coastal environments. Significant results are those with $p$-levels that are less than $3.78 \times 10^{-4}$.

\begin{tabular}{|c|c|c|c|c|}
\hline Analysis No. & Comparison & Inland & Coastal & $p$-value \\
\hline \multirow[t]{5}{*}{$1^{*}$} & Body fossil individuals: $T=1360$ & $\mathrm{~T}=1226$ & $\mathrm{~T}=134$ & $<1 \times 10^{-5}$ \\
\hline & Non-titanosaurs: $\mathrm{T}=987$ & $\mathrm{O}=864$ & $\mathrm{O}=123$ & \\
\hline & & $\mathrm{E}=889.75$ & $\mathrm{E}=97.25$ & \\
\hline & Titanosaurs: $\mathrm{T}=373$ & $\mathrm{O}=362$ & $\mathrm{O}=11$ & \\
\hline & & $E=336.25$ & $E=36.75$ & \\
\hline \multirow[t]{5}{*}{$2^{*}$} & Trackway individuals: $\mathrm{T}=633$ & $\mathrm{~T}=236$ & $\mathrm{~T}=397$ & $<1 \times 10^{-5}$ \\
\hline & Narrow-gauge: $\mathrm{T}=187$ & $\mathrm{O}=30$ & $\mathrm{O}=157$ & \\
\hline & & $\mathrm{E}=69.72$ & $\mathrm{E}=117.28$ & \\
\hline & Wide-gauge: $\mathrm{T}=446$ & $\mathrm{O}=206$ & $\mathrm{O}=240$ & \\
\hline & & $E=166.28$ & $E=279.72$ & \\
\hline \multirow[t]{5}{*}{3} & Body fossil and trackway individuals: $\mathrm{T}=1993$ & $\mathrm{~T}=1462$ & $\mathrm{~T}=531$ & $7 \times 10^{-4}$ \\
\hline & Non-titanosaurs and narrow-gauge: $\mathrm{T}=1174$ & $\mathrm{O}=894$ & $\mathrm{O}=280$ & \\
\hline & & $\mathrm{E}=861.21$ & $E=312.79$ & \\
\hline & Titanosaurs and wide-gauge: $\mathrm{T}=819$ & $\mathrm{O}=568$ & $\mathrm{O}=251$ & \\
\hline & & $\mathrm{E}=600.79$ & $\mathrm{E}=218.21$ & \\
\hline \multirow[t]{5}{*}{$4^{*}$} & Body fossil localities: $\mathrm{T}=706$ & $\mathrm{~T}=624$ & $\mathrm{~T}=82$ & $<1 \times 10^{-5}$ \\
\hline & Non-titanosaurs: $\mathrm{T}=479$ & $\mathrm{O}=404$ & $\mathrm{O}=75$ & \\
\hline & & $E=423.37$ & $\mathrm{E}=55.63$ & \\
\hline & Titanosaurs: $\mathrm{T}=237$ & $\mathrm{O}=227$ & $\mathrm{O}=10$ & \\
\hline & & $E=209.47$ & $\mathrm{E}=27.53$ & \\
\hline \multirow[t]{5}{*}{$5^{*}$} & Trackway localities: $\mathrm{T}=190$ & $\mathrm{~T}=116$ & $\mathrm{~T}=74$ & $8 \times 10^{-5}$ \\
\hline & Narrow-gauge: $\mathrm{T}=41$ & $\mathrm{O}=14$ & $\mathrm{O}=27$ & \\
\hline & & $\mathrm{E}=25.03$ & $\mathrm{E}=15.97$ & \\
\hline & Wide-gauge: $\mathrm{T}=150$ & $\mathrm{O}=102$ & $\mathrm{O}=48$ & \\
\hline & & $\mathrm{E}=91.58$ & $\mathrm{E}=58.42$ & \\
\hline \multirow[t]{5}{*}{6} & Body fossil and trackway localities: $\mathrm{T}=896$ & $\mathrm{~T}=740$ & $\mathrm{~T}=156$ & 0.0677 \\
\hline & Non-titanosaurs and narrow-gauge: $\mathrm{T}=520$ & $\mathrm{O}=418$ & $\mathrm{O}=102$ & \\
\hline & & $\mathrm{E}=429.46$ & $\mathrm{E}=90.54$ & \\
\hline & Titanosaurs and wide-gauge: $\mathrm{T}=387$ & $\mathrm{O}=329$ & $\mathrm{O}=58$ & \\
\hline & & $E=319.62$ & $\mathrm{E}=67.38$ & \\
\hline \multirow[t]{5}{*}{7} & Body fossil individuals: $\mathrm{T}=1360$ & $\mathrm{~T}=1226$ & $\mathrm{~T}=134$ & 0.2145 \\
\hline & Non-titanosauriforms: $\mathrm{T}=819$ & $\mathrm{O}=745$ & $\mathrm{O}=74$ & \\
\hline & & $E=738.30$ & $\mathrm{E}=80.70$ & \\
\hline & Titanosauriforms: $\mathrm{T}=541$ & $\mathrm{O}=481$ & $\mathrm{O}=60$ & \\
\hline & & $\mathrm{E}=487.70$ & $\mathrm{E}=53.30$ & \\
\hline \multirow[t]{5}{*}{$8 \# \#$} & Body fossil and trackway individuals: $\mathrm{T}=1993$ & $\mathrm{~T}=1462$ & $\mathrm{~T}=531$ & $2 \times 10^{-4}$ \\
\hline & Non-titanosauriforms and narrow-gauge: $\mathrm{T}=1006$ & $\mathrm{O}=775$ & $\mathrm{O}=231$ & \\
\hline & & $\mathrm{E}=737.97$ & $E=268.03$ & \\
\hline & Titanosauriforms and wide-gauge: $\mathrm{T}=987$ & $\mathrm{O}=687$ & $\mathrm{O}=300$ & \\
\hline & & $\mathrm{E}=724.03$ & $E=262.97$ & \\
\hline \multirow[t]{5}{*}{9} & Body fossil localities: $\mathrm{T}=706$ & $\mathrm{~T}=624$ & $\mathrm{~T}=82$ & 0.1296 \\
\hline & Non-titanosauriforms: $\mathrm{T}=370$ & $\mathrm{O}=323$ & $\mathrm{O}=47$ & \\
\hline & & $E=327.03$ & $\mathrm{E}=42.97$ & \\
\hline & Titanosauriforms: $\mathrm{T}=359$ & $\mathrm{O}=309$ & $\mathrm{O}=50$ & \\
\hline & & $\mathrm{E}=317.30$ & $\mathrm{E}=41.70$ & \\
\hline \multirow[t]{5}{*}{10} & Body fossil and trackway localities: $\mathrm{T}=896$ & $\mathrm{~T}=740$ & $\mathrm{~T}=156$ & 0.2537 \\
\hline & Non-titanosauriforms and narrow-gauge: $\mathrm{T}=411$ & $\mathrm{O}=337$ & $\mathrm{O}=74$ & \\
\hline & & $\mathrm{E}=339.44$ & $\mathrm{E}=71.56$ & \\
\hline & Titanosauriforms and wide-gauge: $\mathrm{T}=509$ & $\mathrm{O}=411$ & $\mathrm{O}=98$ & \\
\hline & & $\mathrm{E}=420.38$ & $\mathrm{E}=88.62$ & \\
\hline \multirow[t]{5}{*}{$11^{*}$} & Body fossil individuals: $\mathrm{T}=541$ & $\mathrm{~T}=481$ & $\mathrm{~T}=60$ & $<1 \times 10^{-5}$ \\
\hline & Non-titanosaurian titanosauriforms: $\mathrm{T}=168$ & $\mathrm{O}=119$ & $\mathrm{O}=49$ & \\
\hline & & $E=149.37$ & $\mathrm{E}=18.63$ & \\
\hline & Titanosaurs: $\mathrm{T}=373$ & $\mathrm{O}=362$ & $\mathrm{O}=11$ & \\
\hline & & $E=331.63$ & $\mathrm{E}=41.37$ & \\
\hline
\end{tabular}


TABle 2. Continued.

\begin{tabular}{|c|c|c|c|c|}
\hline Analysis No. & Comparison & Inland & Coastal & $p$-value \\
\hline $12^{*}$ & $\begin{array}{l}\text { Body fossil localities: } \mathrm{T}=362 \\
\text { Non-titanosaurian titanosauriforms: } \mathrm{T}=122 \\
\text { Titanosaurs: } \mathrm{T}=237\end{array}$ & $\begin{aligned} \mathrm{T} & =310 \\
\mathrm{O} & =82 \\
\mathrm{E} & =104.48 \\
\mathrm{O} & =227 \\
\mathrm{E} & =202.96\end{aligned}$ & $\begin{aligned} \mathrm{T} & =52 \\
\mathrm{O} & =40 \\
\mathrm{E} & =17.52 \\
\mathrm{O} & =10 \\
\mathrm{E} & =34.04\end{aligned}$ & $<1 \times 10^{-5}$ \\
\hline 13 & $\begin{array}{l}\text { Body fossil individuals: } \mathrm{T}=1360 \\
\text { Non-macronarians: } \mathrm{T}=565 \\
\text { Macronarians: } \mathrm{T}=795\end{array}$ & $\begin{aligned} \mathrm{T} & =1225 \\
\mathrm{O} & =509 \\
\mathrm{E} & =508.92 \\
\mathrm{O} & =716 \\
\mathrm{E} & =716.08\end{aligned}$ & $\begin{array}{l}\mathrm{T}=135 \\
\mathrm{O}=56 \\
\mathrm{E}=56.08 \\
\mathrm{O}=79 \\
\mathrm{E}=78.92\end{array}$ & 0.9383 \\
\hline 14 & $\begin{array}{l}\text { Body fossil localities: } \mathrm{T}=706 \\
\text { Non-macronarians: } \mathrm{T}=307 \\
\text { Macronarians: } \mathrm{T}=467\end{array}$ & $\begin{aligned} \mathrm{T} & =624 \\
\mathrm{O} & =268 \\
\mathrm{E} & =271.34 \\
\mathrm{O} & =412 \\
\mathrm{E} & =412.76\end{aligned}$ & $\begin{array}{l}\mathrm{T}=82 \\
\mathrm{O}=39 \\
\mathrm{E}=35.66 \\
\mathrm{O}=55 \\
\mathrm{E}=54.24\end{array}$ & 0.5435 \\
\hline
\end{tabular}

body fossil data set to Titanosauriformes, partitioning the data into titanosaurs and non-titanosaurs (Analyses 11 and 12, Table 2). Both analyses display strong support $(p<1 \times$ $10^{-5}$ ) for titanosaurs occurring more often than expected in inland environments, and basal titanosauriforms occurring more often than expected in coastal ones.

Macronarians versus Non-Macronarians.Analyses 13 and 14 (Table 2) compare the distributions of Macronaria and non-Macronaria for all sauropods. Both analyses fail the chi-square test (Table 2), indicating that there is no significant support for environmental associations when sauropods are partitioned into Macronaria versus non-Macronaria (contra Butler and Barrett 2008; see below).

Analyses 15-115: Time-Slicing the Data

Cretaceous Time Slices.-Analyses 15-17 (Table 3) repeat Analyses 1, 5, and 7 respectively, but are restricted to Cretaceous sauropods alone. Analysis 15 passes the chisquare tests $\left(p<1 \times 10^{-5}\right)$, demonstrating that the restriction of the data set does not affect conclusions based on Analysis 1. The previous positive association between widegauge trackways and inland environments and narrow-gauge trackways and coastal environments (Analysis 5) disappears when only Cretaceous data are examined (Analysis 16), probably because there are very few narrow-gauge trackways known from the Cretaceous (six in our data set; see Supplementary Materials). Analysis 7 failed the chi- square test, but Analysis 17 yields a positive association between titanosauriforms and inland environments and non-titanosauriforms and coastal environments. This result probably occurs because restricting "Titanosauriformes" to Cretaceous taxa alone raises the proportion of titanosaurs.

Analyse 18 and 19 examine the distributions of Macronaria and non-Macronaria based on just the Cretaceous part of our data set. As before there is no significant support for environmental associations when sauropods are partitioned into these two groups, although the $p$-values are substantially lower $(p<0.14)$ than for when all sauropods are considered (Analyses 13 and 14, $p>0.5$ ). This phenomenon seems to occur because the taxonomic content of "Cretaceous Macronaria" is very similar to that of Titanosauriformes and Titanosauria.

Analysis of Butler and Barrett's (2008) data set of Cretaceous sauropods (Analyses 20-23) uses another time slice of the total available data to examine environmental associations between titanosaurs and non-titanosaurs, and between titanosauriforms and non-titanosauriforms. Although results are consistent with positive associations between titanosaurs/titanosauriforms and inland environments, none pass the chi-square test $(p>$ 0.0017, Table 3).

Within-Cretaceous Time Slices.-Analyses 24-29 (Table 3) repeat Analyses 1-6, but with the data restricted to the Early and early Late Cretaceous (Berriasian-Coniacian). Analyses 
TABLE 3. Summary of analyses (15-29) of potential environmental associations among sauropods, based on larger time slices such as "Cretaceous," "Early Cretaceous," etc. (see "Analyses and Results" for details). For abbreviations and symbols, see legend to Table 2.

\begin{tabular}{|c|c|c|c|c|}
\hline Analysis no. & Comparison & Inland & Coastal & $p$-value \\
\hline \multirow[t]{5}{*}{$15^{*}$} & Body fossil individuals (Cretaceous only): $\mathrm{T}=526$ & $\mathrm{~T}=499$ & $\mathrm{~T}=27$ & $<1 \times 10^{-5}$ \\
\hline & Non-titanosaurs: $\mathrm{T}=152$ & $\mathrm{O}=131$ & $\mathrm{O}=21$ & \\
\hline & & $\mathrm{E}=144.20$ & $\mathrm{E}=7.80$ & \\
\hline & Titanosaurs: $\mathrm{T}=374$ & $\mathrm{O}=368$ & $\mathrm{O}=6$ & \\
\hline & & $\mathrm{E}=354.80$ & $\mathrm{E}=19.20$ & \\
\hline \multirow[t]{4}{*}{16} & Trackway localities (Cretaceous only): $\mathrm{T}=131$ & $\mathrm{~T}=97$ & $\mathrm{~T}=34$ & 0.6748 \\
\hline & Narrow-gauge: $\mathrm{T}=6$ & $\mathrm{O}=4$ & $\mathrm{O}=2$ & \\
\hline & Wide-gauge: $T=125$ & $\begin{aligned} E & =4.44 \\
O & =93\end{aligned}$ & $\begin{aligned} E & =1.56 \\
O & =32\end{aligned}$ & \\
\hline & & $\mathrm{E}=92.56$ & $\mathrm{E}=32.44$ & \\
\hline \multirow[t]{5}{*}{$17 \#$} & Body fossil individuals (Cretaceous only): $\mathrm{T}=526$ & $\mathrm{~T}=499$ & $\mathrm{~T}=27$ & $<1 \times 10^{-5}$ \\
\hline & Non-titanosauriforms: $\mathrm{T}=65$ & $\mathrm{O}=54$ & $\mathrm{O}=11$ & \\
\hline & & $E=61.66$ & $\mathrm{E}=3.34$ & \\
\hline & Titanosauriforms: $\mathrm{T}=461$ & $\mathrm{O}=445$ & $\mathrm{O}=16$ & \\
\hline & & $E=437.34$ & $E=23.66$ & \\
\hline \multirow[t]{4}{*}{18} & Body fossil localities (Cretaceous only): $\mathrm{T}=525$ & $\mathrm{~T}=496$ & $\mathrm{~T}=29$ & 0.0574 \\
\hline & Non-macronarians: $\mathrm{T}=54$ & $\begin{aligned} \mathrm{O} & =48 \\
\mathrm{E} & =51.02\end{aligned}$ & $\begin{array}{l}\mathrm{O}=6 \\
\mathrm{E}=2.98\end{array}$ & \\
\hline & Macronarians: $\mathrm{T}=471$ & $\mathrm{O}=448$ & $\mathrm{O}=23$ & \\
\hline & & $\mathrm{E}=444.98$ & $\mathrm{E}=26.02$ & \\
\hline \multirow[t]{5}{*}{19} & Body fossil localities (Cretaceous only): $\mathrm{T}=334$ & $\mathrm{~T}=312$ & $\mathrm{~T}=22$ & 0.1351 \\
\hline & Non-macronarians: $\mathrm{T}=41$ & $\mathrm{O}=36$ & $\mathrm{O}=5$ & \\
\hline & & $E=38.30$ & $\mathrm{E}=2.70$ & \\
\hline & Macronarians: $\mathrm{T}=297$ & $\mathrm{O}=279$ & $\mathrm{O}=18$ & \\
\hline & & $\mathrm{E}=277.44$ & $E=19.56$ & \\
\hline \multirow[t]{5}{*}{20} & $\begin{array}{l}\text { Body fossil localities (Butler and Barrett Cretaceous } \\
\text { data): } \mathrm{T}=175\end{array}$ & $\mathrm{~T}=166$ & $\mathrm{~T}=9$ & 0.0019 \\
\hline & Non-titanosaurs: $\mathrm{T}=78$ & $\mathrm{O}=69$ & $\mathrm{O}=9$ & \\
\hline & & $E=73.99$ & $\mathrm{E}=4.01$ & \\
\hline & Titanosaurs: $\mathrm{T}=123$ & $\mathrm{O}=121$ & $\mathrm{O}=2$ & \\
\hline & & $E=116.67$ & $E=6.33$ & \\
\hline \multirow[t]{5}{*}{21} & $\begin{array}{l}\text { Body fossil and trackway localities (Butler and } \\
\text { Barrett Cretaceous data): } T=177\end{array}$ & $\mathrm{~T}=168$ & $\mathrm{~T}=9$ & 0.0017 \\
\hline & Non-titanosaurs and narrow-gauge: $\mathrm{T}=78$ & $\mathrm{O}=69$ & $\mathrm{O}=9$ & \\
\hline & & $\mathrm{E}=74.03$ & $\mathrm{E}=3.97$ & \\
\hline & Titanosaurs and wide-gauge: $\mathrm{T}=125$ & $\mathrm{O}=123$ & $\mathrm{O}=2$ & \\
\hline & & $E=118.64$ & $E=6.36$ & \\
\hline \multirow[t]{5}{*}{22} & $\begin{array}{l}\text { Body fossil localities (Butler and Barrett Cretaceous } \\
\text { data): } \mathrm{T}=175\end{array}$ & $\mathrm{~T}=166$ & $\mathrm{~T}=9$ & 0.0178 \\
\hline & Non-titanosauriforms: $\mathrm{T}=27$ & $\mathrm{O}=23$ & $\mathrm{O}=4$ & \\
\hline & & $E=25.61$ & $\mathrm{E}=1.39$ & \\
\hline & Titanosauriforms: $\mathrm{T}=174$ & $\mathrm{O}=167$ & $\mathrm{O}=7$ & \\
\hline & & $\mathrm{E}=165.05$ & $\mathrm{E}=8.95$ & \\
\hline \multirow[t]{5}{*}{23} & $\begin{array}{l}\text { Body fossil and trackway localities (Butler and } \\
\text { Barrett Cretaceous data): } \mathrm{T}=177\end{array}$ & $\mathrm{~T}=168$ & $\mathrm{~T}=9$ & 0.0163 \\
\hline & Non-titanosauriforms and narrow-gauge: $\mathrm{T}=27$ & $\mathrm{O}=23$ & $\mathrm{O}=4$ & \\
\hline & & $E=25.63$ & $\mathrm{E}=1.37$ & \\
\hline & Titanosauriforms and wide-gauge: $\mathrm{T}=176$ & $\mathrm{O}=169$ & $\mathrm{O}=7$ & \\
\hline & & $E=167.05$ & $\mathrm{E}=8.95$ & \\
\hline \multirow[t]{5}{*}{$24^{*}$} & $\begin{array}{l}\text { Body fossil individuals (Early-mid Cretaceous only): } \\
\quad \mathrm{T}=259\end{array}$ & $\mathrm{~T}=237$ & $\mathrm{~T}=22$ & $1 \times 10^{-4}$ \\
\hline & Non-titanosaurs: $\mathrm{T}=135$ & $\mathrm{O}=115$ & $\mathrm{O}=20$ & \\
\hline & & $E=123.53$ & $\mathrm{E}=11.47$ & \\
\hline & Titanosaurs: $\mathrm{T}=124$ & $\mathrm{O}=122$ & $\mathrm{O}=2$ & \\
\hline & & $\mathrm{E}=113.47$ & $\mathrm{E}=10.53$ & \\
\hline \multirow[t]{5}{*}{25} & $\begin{array}{l}\text { Trackway individuals (Early-mid Cretaceous): } \mathrm{T}= \\
\quad 284\end{array}$ & $\mathrm{~T}=180$ & $\mathrm{~T}=104$ & 0.2769 \\
\hline & Narrow-gauge: $\mathrm{T}=3$ & $\mathrm{O}=1$ & $\mathrm{O}=2$ & \\
\hline & & $\mathrm{E}=1.9$ & $\mathrm{E}=1.1$ & \\
\hline & Wide-gauge: $\mathrm{T}=281$ & $\mathrm{O}=179$ & $\mathrm{O}=102$ & \\
\hline & & $\mathrm{E}=178.1$ & $\mathrm{E}=102.9$ & \\
\hline
\end{tabular}


TABle 3. Continued.

\begin{tabular}{|c|c|c|c|c|}
\hline Analysis no. & Comparison & Inland & Coastal & $p$-value \\
\hline \multirow[t]{5}{*}{26} & $\begin{array}{l}\text { Body fossil and trackway individuals (Early-mid } \\
\text { Cretaceous): } \mathrm{T}=543\end{array}$ & $\mathrm{~T}=417$ & $\mathrm{~T}=126$ & 0.0193 \\
\hline & Non-titanosaurs and narrow-gauge: $\mathrm{T}=138$ & $\mathrm{O}=116$ & $\mathrm{O}=22$ & \\
\hline & & $E=105.98$ & $E=32.02$ & \\
\hline & Titanosaurs and wide-gauge: $\mathrm{T}=405$ & $\mathrm{O}=301$ & $\mathrm{O}=104$ & \\
\hline & & $\mathrm{E}=311.02$ & $\mathrm{E}=93.98$ & \\
\hline \multirow[t]{5}{*}{27} & Body fossil localities (Early-mid Cretaceous): $\mathrm{T}=162$ & $\mathrm{~T}=144$ & $\mathrm{~T}=18$ & 0.0039 \\
\hline & Non-titanosaurs: $\mathrm{T}=95$ & $\mathrm{O}=79$ & $\mathrm{O}=16$ & \\
\hline & & $\mathrm{E}=84.44$ & $\mathrm{E}=10.56$ & \\
\hline & Titanosaurs: $\mathrm{T}=73$ & $\mathrm{O}=71$ & $\mathrm{O}=2$ & \\
\hline & & $E=64.89$ & $\mathrm{E}=8.11$ & \\
\hline \multirow[t]{4}{*}{28} & Trackway localities (Early-mid Cretaceous): $\mathrm{T}=120$ & $\mathrm{~T}=88$ & $\mathrm{~T}=32$ & 0.1126 \\
\hline & Narrow-gauge: $\mathrm{T}=3$ & $\begin{aligned} \mathrm{O} & =1 \\
\mathrm{E} & =2.20\end{aligned}$ & $\begin{aligned} \mathrm{O} & =2 \\
\mathrm{E} & =0.80\end{aligned}$ & \\
\hline & Wide-gauge: $\mathrm{T}=117$ & $\mathrm{O}=87$ & $\mathrm{O}=30$ & \\
\hline & & $\mathrm{E}=85.80$ & $\mathrm{E}=31.20$ & \\
\hline \multirow[t]{5}{*}{29} & $\begin{array}{l}\text { Body fossil and trackway localities (Early-mid } \\
\text { Cretaceous): } \mathrm{T}=282\end{array}$ & $\mathrm{~T}=232$ & $\mathrm{~T}=50$ & 0.7195 \\
\hline & Non-titanosaurs and narrow-gauge: $\mathrm{T}=98$ & $\mathrm{O}=80$ & $\mathrm{O}=18$ & \\
\hline & & $\mathrm{E}=80.62$ & $\mathrm{E}=17.38$ & \\
\hline & Titanosaurs and wide-gauge: $\mathrm{T}=190$ & $\mathrm{O}=158$ & $\mathrm{O}=32$ & \\
\hline & & $E=156.31$ & $E=33.69$ & \\
\hline
\end{tabular}

25-29 produce non-significant $p$-values. However, the remaining analysis (no. 24, bodyfossil individuals) still supports the positive association between titanosaurs and inland environments $\left(p=1 \times 10^{-4}\right)$.

Stage-Level Time-Slicing.-Analyses 30-115 (Tables 4-9) replicate Analyses 1-6, but with data restricted to a single European stage of the Jurassic or Cretaceous. Of these 86 analyses, only 22 produced statistically significant results: 13 in which the association between titanosaurs and/or wide gauge trackways and inland environments was negative, and nine in which it was positive. Although these results appear to conflict, it should be noted that 12 of the 13 analyses with "negative" results were of Middle and Late Jurassic stages (the exception is Analysis 66-Albian body fossil and trackway individuals), whereas all nine analyses supporting the positive association with inland environments were of Cretaceous stages. These results reflect the fact that the earliest widegauge trackways (e.g., the Middle Jurassic Ardley site) and the earliest titanosaurian body fossils (Janenschia, from the Late Jurassic of Tendaguru, Tanzania) are preserved in coastal environments (Aberhan et al. 2002;
Day et al. 2002, 2004). This issue will be addressed further in the "Discussion". For now, it is sufficient to note that even when the data set is time-sliced to stage level, many statistically significant environmental associations occur, suggesting that the results cannot be explained merely as an artifact created by uneven sampling of the two environment types from deposits of Jurassic and Cretaceous age.

\section{Analyses 116-134: "Jack-knifing" the Data}

Following exclusion of the Korean tracksites, environmental associations are not supported by analyses 119-121 (Table 10, $p$ $>0.2432$ ), which are based on the number of localities. Analyses 116-118, based on number of individuals, pass the chi-square test $(p<$ $1 \times 10^{-5}$ ). Analysis 116 (based on trackway data alone) continues to support the positive associations between wide-gauge trackways and inland environments and narrow-gauge trackways and coastal environments. However, the addition of the body fossil data in Analyses 117 and 118 reverses the polarity of these associations. Analysis 122 explores what happens when the 73 Korean tracksites are treated as six separate localities, but this 
TABLE 4. Results of Analyses 30-43 (sauropod body fossil individuals) per European stage. Only stages where both titanosaurs and non-titanosaur body fossils are known are shown. See Table 2 for explanation of abbreviations and symbols.

\begin{tabular}{|c|c|c|c|c|c|c|}
\hline \multirow[b]{2}{*}{ Analysis no. } & \multirow[b]{2}{*}{ Stage } & \multicolumn{2}{|c|}{ Non-titanosaurs } & \multicolumn{2}{|c|}{ Titanosaurs } & \multirow[b]{2}{*}{$p$-value } \\
\hline & & Inland & Coastal & Inland & Coastal & \\
\hline $30^{* *}$ & Kimmeridgian & $\begin{aligned} \mathrm{O} & =582 \\
\mathrm{E} & =576.42\end{aligned}$ & $\begin{array}{l}\mathrm{O}=38 \\
\mathrm{E}=43.58\end{array}$ & $\begin{aligned} \mathrm{O} & =0 \\
\mathrm{E} & =5.58\end{aligned}$ & $\begin{array}{l}\mathrm{O}=6 \\
\mathrm{E}=0.42\end{array}$ & $<1 \times 10^{-5}$ \\
\hline $31^{* *}$ & Tithonian & $\begin{array}{l}\mathrm{O}=489 \\
\mathrm{E}=482.10\end{array}$ & $\begin{array}{l}\mathrm{O}=70 \\
\mathrm{E}=76.90\end{array}$ & $\begin{array}{l}\mathrm{O}=0 \\
\mathrm{E}=6.90\end{array}$ & $\begin{array}{l}\mathrm{E}=0.42 \\
\mathrm{O}=8 \\
\mathrm{E}=1.10\end{array}$ & $<1 \times 10^{-5}$ \\
\hline 32 & Berriasian & $\begin{array}{l}\mathrm{O}=20 \\
\mathrm{E}=20.00\end{array}$ & $\begin{array}{l}\mathrm{O}=0 \\
\mathrm{E}=0\end{array}$ & $\begin{array}{l}\mathrm{O}=6 \\
\mathrm{E}=6.00\end{array}$ & $\begin{array}{l}\mathrm{O}=0 \\
\mathrm{E}=0\end{array}$ & 1 \\
\hline 33 & Valanginian & $\begin{array}{l}\mathrm{O}=13 \\
\mathrm{E}=13.00\end{array}$ & $\begin{array}{l}\mathrm{O}=0 \\
\mathrm{E}=0\end{array}$ & $\begin{aligned} \mathrm{O} & =19 \\
\mathrm{E} & =19.00\end{aligned}$ & $\begin{array}{l}\mathrm{O}=0 \\
\mathrm{E}=0\end{array}$ & 1 \\
\hline 34 & Hauterivian & $\begin{array}{l}\mathrm{O}=35 \\
\mathrm{E}=36.36\end{array}$ & $\begin{array}{l}\mathrm{O}=4 \\
\mathrm{E}=2.64\end{array}$ & $\begin{aligned} \mathrm{O} & =20 \\
\mathrm{E} & =18.64\end{aligned}$ & $\begin{array}{l}\mathrm{O}=0 \\
\mathrm{E}=1.36\end{array}$ & 0.1371 \\
\hline 35 & Barremian & $\begin{array}{l}\mathrm{O}=52 \\
\mathrm{E}=56.38\end{array}$ & $\begin{array}{l}\mathrm{O}=13 \\
\mathrm{E}=8.62\end{array}$ & $\begin{array}{l}\mathrm{O}=33 \\
\mathrm{E}=28.62\end{array}$ & $\begin{array}{l}\mathrm{O}=0 \\
\mathrm{E}=4.38\end{array}$ & 0.0058 \\
\hline 36 & Aptian & $\begin{aligned} \mathrm{O} & =50 \\
\mathrm{E} & =52.00\end{aligned}$ & $\begin{array}{l}\mathrm{O}=6 \\
\mathrm{E}=4.00\end{array}$ & $\begin{aligned} \mathrm{O} & =28 \\
\mathrm{E} & =26.00\end{aligned}$ & $\begin{array}{l}\mathrm{O}=0 \\
\mathrm{E}=2.00\end{array}$ & 0.0723 \\
\hline 37 & Albian & $\begin{array}{l}\mathrm{O}=61 \\
\mathrm{E}=62.23\end{array}$ & $\begin{array}{l}\mathrm{O}=4 \\
\mathrm{E}=2.77\end{array}$ & $\begin{aligned} \mathrm{O} & =29 \\
\mathrm{E} & =27.77\end{aligned}$ & $\begin{array}{l}\mathrm{O}=0 \\
\mathrm{E}=1.23\end{array}$ & 0.1733 \\
\hline 38 & Cenomanian & $\begin{array}{l}\mathrm{O}=23 \\
\mathrm{E}=23.21\end{array}$ & $\begin{array}{l}\mathrm{O}=1 \\
\mathrm{E}=0.79\end{array}$ & $\begin{array}{l}\mathrm{O}=36 \\
\mathrm{E}=35.79\end{array}$ & $\begin{array}{l}\mathrm{O}=1 \\
\mathrm{E}=1.21\end{array}$ & 0.7567 \\
\hline 39 & Turonian & $\begin{array}{l}\mathrm{O}=11 \\
\mathrm{E}=11.00\end{array}$ & $\begin{array}{l}\mathrm{O}=0 \\
\mathrm{E}=0\end{array}$ & $\begin{array}{l}\mathrm{O}=52 \\
\mathrm{E}=52.00\end{array}$ & $\begin{array}{l}\mathrm{O}=0 \\
\mathrm{E}=0\end{array}$ & 1 \\
\hline 40 & Coniacian & $\begin{aligned} \mathrm{O} & =5 \\
\mathrm{E} & =5.00\end{aligned}$ & $\begin{array}{l}\mathrm{O}=0 \\
\mathrm{E}=0\end{array}$ & $\begin{aligned} \mathrm{O} & =51 \\
\mathrm{E} & =51.00\end{aligned}$ & $\begin{array}{l}\mathrm{O}=0 \\
\mathrm{E}=0\end{array}$ & 1 \\
\hline 41 & Santonian & $\begin{array}{l}\mathrm{O}=3 \\
\mathrm{E}=3.00\end{array}$ & $\begin{array}{l}\mathrm{O}=0 \\
\mathrm{E}=0\end{array}$ & $\begin{array}{l}\mathrm{O}=46 \\
\mathrm{E}=46.00\end{array}$ & $\begin{array}{l}\mathrm{O}=0 \\
\mathrm{E}=0\end{array}$ & 1 \\
\hline 42 & Campanian & $\begin{array}{l}\mathrm{O}=4 \\
\mathrm{E}=3.94\end{array}$ & $\begin{array}{l}\mathrm{O}=0 \\
\mathrm{E}=0.06\end{array}$ & $\begin{array}{l}O=126 \\
E=126.06\end{array}$ & $\begin{array}{l}\mathrm{O}=2 \\
\mathrm{E}=1.94\end{array}$ & 0.8018 \\
\hline $43^{*}$ & Maastrichtian & $\begin{array}{l}\mathrm{O}=5 \\
\mathrm{E}=9.75\end{array}$ & $\begin{array}{l}\mathrm{O}=5 \\
\mathrm{E}=0.25\end{array}$ & $\begin{array}{l}\mathrm{O}=189 \\
\mathrm{E}=184.25\end{array}$ & $\begin{array}{l}\mathrm{O}=0 \\
\mathrm{E}=4.75\end{array}$ & $<1 \times 10^{-5}$ \\
\hline
\end{tabular}

TABle 5. Results of Analyses 44-55 (sauropod tracksite individuals) per European stage. Only stages where both titanosaurs and non-titanosaurs are known are shown. See Table 2 for explanation of abbreviations and symbols.

\begin{tabular}{|c|c|c|c|c|c|c|}
\hline \multirow[b]{2}{*}{ Analysis no. } & \multirow[b]{2}{*}{ Stage } & \multicolumn{2}{|c|}{ Narrow-gauge } & \multicolumn{2}{|c|}{ Wide-gauge } & \multirow[b]{2}{*}{$p$-value } \\
\hline & & Inland & Coastal & Inland & Coastal & \\
\hline $44^{* *}$ & Bajocian & $\begin{array}{l}\mathrm{O}=8 \\
\mathrm{E}=3.56\end{array}$ & $\begin{array}{l}\mathrm{O}=4 \\
\mathrm{E}=8.44\end{array}$ & $\begin{array}{l}\mathrm{O}=0 \\
\mathrm{E}=4.44\end{array}$ & $\begin{aligned} \mathrm{O} & =15 \\
\mathrm{E} & =10.56\end{aligned}$ & $2 \times 10^{-4}$ \\
\hline $45^{* *}$ & Bathonian & $\begin{array}{l}\mathrm{O}=17 \\
\mathrm{E}=9.83\end{array}$ & $\begin{array}{l}\mathrm{O}=31 \\
\mathrm{E}=38.17\end{array}$ & $\begin{array}{l}\mathrm{O}=0 \\
\mathrm{E}=7.17\end{array}$ & $\begin{array}{l}\mathrm{O}=35 \\
\mathrm{E}=27.83\end{array}$ & $8 \times 10^{-5}$ \\
\hline 46 & Callovian & $\begin{aligned} \mathrm{O} & =8 \\
\mathrm{E} & =8.31\end{aligned}$ & $\begin{array}{l}\mathrm{O}=4 \\
\mathrm{E}=3.69\end{array}$ & $\begin{array}{l}\mathrm{O}=1 \\
\mathrm{E}=0.69\end{array}$ & $\begin{array}{l}\mathrm{O}=0 \\
\mathrm{E}=0.31\end{array}$ & 0.4857 \\
\hline 47 & Kimmeridgian & $\begin{aligned} \mathrm{O} & =8 \\
\mathrm{E} & =12.36\end{aligned}$ & $\begin{aligned} \mathrm{O} & =70 \\
\mathrm{E} & =65.64\end{aligned}$ & $\begin{array}{l}\mathrm{O}=8 \\
\mathrm{E}=3.64\end{array}$ & $\begin{array}{l}\mathrm{O}=15 \\
\mathrm{E}=19.36\end{array}$ & 0.0046 \\
\hline 48 & Tithonian & $\begin{array}{l}\mathrm{O}=3 \\
\mathrm{E}=7.33\end{array}$ & $\begin{array}{l}\mathrm{O}=91 \\
\mathrm{E}=86.67\end{array}$ & $\begin{array}{l}\mathrm{O}=8 \\
\mathrm{E}=3.67\end{array}$ & $\begin{array}{l}\mathrm{O}=39 \\
\mathrm{E}=43.33\end{array}$ & 0.0039 \\
\hline 49 & Berriasian & $\begin{aligned} \mathrm{O} & =0 \\
\mathrm{E} & =0.79\end{aligned}$ & $\begin{aligned} \mathrm{O} & =1 \\
\mathrm{E} & =0.21\end{aligned}$ & $\begin{array}{l}\mathrm{O}=34 \\
\mathrm{E}=33.21\end{array}$ & $\begin{aligned} \mathrm{O} & =8 \\
\mathrm{E} & =8.79\end{aligned}$ & 0.0497 \\
\hline $50^{*}$ & Valanginian & $\begin{aligned} \mathrm{O} & =0 \\
\mathrm{E} & =0.94\end{aligned}$ & $\begin{array}{l}\mathrm{O}=1 \\
\mathrm{E}=0.06\end{array}$ & $\begin{array}{l}\mathrm{O}=32 \\
\mathrm{E}=31.06\end{array}$ & $\begin{array}{l}\mathrm{O}=1 \\
\mathrm{E}=1.94\end{array}$ & $6 \times 10^{-5}$ \\
\hline $51^{*}$ & Hauterivian & $\begin{array}{l}\mathrm{O}=0 \\
\mathrm{E}=0.94\end{array}$ & $\begin{array}{l}\mathrm{O}=1 \\
\mathrm{E}=0.06\end{array}$ & $\begin{array}{l}\mathrm{O}=32 \\
\mathrm{E}=31.06\end{array}$ & $\begin{array}{l}\mathrm{O}=1 \\
\mathrm{E}=1.94\end{array}$ & $6 \times 10^{-5}$ \\
\hline $52^{*}$ & Barremian & $\begin{array}{l}\mathrm{O}=0 \\
\mathrm{E}=0.98\end{array}$ & $\begin{array}{l}\mathrm{O}=1 \\
\mathrm{E}=0.02\end{array}$ & $\begin{array}{l}\mathrm{O}=41 \\
\mathrm{E}=40.02\end{array}$ & $\begin{aligned} \mathrm{O} & =0 \\
\mathrm{E} & =0.98\end{aligned}$ & $<1 \times 10^{-5}$ \\
\hline $53^{*}$ & Aptian & $\begin{array}{l}\mathrm{O}=0 \\
\mathrm{E}=0.98\end{array}$ & $\begin{array}{l}\mathrm{O}=1 \\
\mathrm{E}=0.02\end{array}$ & $\begin{array}{l}\mathrm{O}=100 \\
\mathrm{E}=99.02\end{array}$ & $\begin{aligned} \mathrm{O} & =1 \\
\mathrm{E} & =0.98\end{aligned}$ & $<1 \times 10^{-5}$ \\
\hline 54 & Albian & $\begin{aligned} \mathrm{O} & =1 \\
\mathrm{E} & =0.62\end{aligned}$ & $\begin{array}{l}\mathrm{O}=0 \\
\mathrm{E}=0.38\end{array}$ & $\begin{aligned} \mathrm{O} & =137 \\
\mathrm{E} & =137.38\end{aligned}$ & $\begin{aligned} \mathrm{O} & =86 \\
\mathrm{E} & =85.62\end{aligned}$ & 0.4337 \\
\hline 55 & Campanian & $\begin{array}{l}\mathrm{O}=3 \\
\mathrm{E}=1.83\end{array}$ & $\begin{array}{l}\mathrm{O}=0 \\
\mathrm{E}=1.17\end{array}$ & $\begin{array}{l}\mathrm{O}=11 \\
\mathrm{E}=12.17\end{array}$ & $\begin{aligned} \mathrm{O} & =9 \\
\mathrm{E} & =7.83\end{aligned}$ & 0.1376 \\
\hline
\end{tabular}


TABLE 6. Results of Analyses 56-72 (sauropod body fossil and tracksite individuals) per European stage. Only stages where both titanosaurs and non-titanosaurs are known are shown. See Table 2 for explanation of abbreviations and symbols.

\begin{tabular}{|c|c|c|c|c|c|c|}
\hline \multirow[b]{2}{*}{ Analysis no. } & \multirow[b]{2}{*}{ Stage } & \multicolumn{2}{|c|}{ Non-titanosaurs and narrow-gauge } & \multicolumn{2}{|c|}{ Titanosaurs and wide-gauge } & \multirow[b]{2}{*}{$p$-value } \\
\hline & & Inland & Coastal & Inland & Coastal & \\
\hline $56^{* *}$ & Bajocian & $\begin{array}{l}\mathrm{O}=52 \\
\mathrm{E}=41.01\end{array}$ & $\begin{array}{l}\mathrm{O}=4 \\
\mathrm{E}=14.99\end{array}$ & $\begin{aligned} \mathrm{O} & =0 \\
\mathrm{E} & =10.99\end{aligned}$ & $\begin{array}{l}\mathrm{O}=15 \\
\mathrm{E}=4.01\end{array}$ & $<1 \times 10^{-5}$ \\
\hline $57^{* *}$ & Bathonian & $\begin{aligned} \mathrm{O} & =90 \\
\mathrm{E} & =70.06\end{aligned}$ & $\begin{aligned} \mathrm{O} & =33 \\
\mathrm{E} & =52.94\end{aligned}$ & $\begin{aligned} \mathrm{O} & =0 \\
\mathrm{E} & =19.94\end{aligned}$ & $\begin{aligned} \mathrm{O} & =35 \\
\mathrm{E} & =15.06\end{aligned}$ & $<1 \times 10^{-5}$ \\
\hline 58 & Callovian & $\begin{array}{l}\mathrm{O}=89 \\
\mathrm{E}=89.04\end{array}$ & $\begin{array}{l}\mathrm{O}=4 \\
\mathrm{E}=3.96\end{array}$ & $\begin{array}{l}\mathrm{O}=1 \\
\mathrm{E}=0.96\end{array}$ & $\begin{array}{l}\mathrm{O}=0 \\
\mathrm{E}=0.04\end{array}$ & 0.8376 \\
\hline $59^{* *}$ & Kimmeridgian & $\begin{array}{l}\mathrm{O}=590 \\
\mathrm{E}=574.15\end{array}$ & $\begin{array}{l}\mathrm{O}=108 \\
\mathrm{E}=123.85\end{array}$ & $\begin{aligned} \mathrm{O} & =8 \\
\mathrm{E} & =23.85\end{aligned}$ & $\begin{array}{l}\mathrm{O}=21 \\
\mathrm{E}=5.15\end{array}$ & $<1 \times 10^{-5}$ \\
\hline $60^{* *}$ & Tithonian & $\begin{array}{l}\mathrm{O}=492 \\
\mathrm{E}=461.16\end{array}$ & $\begin{array}{l}\mathrm{O}=161 \\
\mathrm{E}=191.84\end{array}$ & $\begin{aligned} \mathrm{O} & =8 \\
\mathrm{E} & =33.84\end{aligned}$ & $\begin{array}{l}\mathrm{O}=47 \\
\mathrm{E}=16.16\end{array}$ & $<1 \times 10^{-5}$ \\
\hline 61 & Berriasian & $\begin{array}{l}\mathrm{O}=20 \\
\mathrm{E}=18.26\end{array}$ & $\begin{array}{l}\mathrm{O}=1 \\
\mathrm{E}=2.74\end{array}$ & $\begin{aligned} \mathrm{O} & =40 \\
\mathrm{E} & =41.74\end{aligned}$ & $\begin{array}{l}\mathrm{O}=8 \\
\mathrm{E}=6.26\end{array}$ & 0.1416 \\
\hline 62 & Valanginian & $\begin{array}{l}\mathrm{O}=13 \\
\mathrm{E}=13.58\end{array}$ & $\begin{array}{l}\mathrm{O}=1 \\
\mathrm{E}=0.42\end{array}$ & $\begin{aligned} \mathrm{O} & =51 \\
\mathrm{E} & =50.42\end{aligned}$ & $\begin{aligned} \mathrm{O} & =1 \\
\mathrm{E} & =1.58\end{aligned}$ & 0.3078 \\
\hline 63 & Hauterivian & $\begin{aligned} \mathrm{O} & =35 \\
\mathrm{E} & =37.42\end{aligned}$ & $\begin{array}{l}\mathrm{O}=5 \\
\mathrm{E}=2.58\end{array}$ & $\begin{aligned} \mathrm{O} & =52 \\
\mathrm{E} & =49.58\end{aligned}$ & $\begin{aligned} \mathrm{O} & =1 \\
\mathrm{E} & =3.42\end{aligned}$ & 0.0391 \\
\hline $64^{*}$ & Barremian & $\begin{array}{l}\mathrm{O}=52 \\
\mathrm{E}=59.40\end{array}$ & $\begin{array}{l}\mathrm{O}=14 \\
\mathrm{E}=6.60\end{array}$ & $\begin{array}{l}\mathrm{O}=74 \\
\mathrm{E}=66.60\end{array}$ & $\begin{array}{l}\mathrm{O}=0 \\
\mathrm{E}=7.40\end{array}$ & $3 \times 10^{-5}$ \\
\hline $65^{*}$ & Aptian & $\begin{array}{l}\mathrm{O}=50 \\
\mathrm{E}=54.55\end{array}$ & $\begin{array}{l}\mathrm{O}=7 \\
\mathrm{E}=2.45\end{array}$ & $\begin{array}{l}\mathrm{O}=128 \\
\mathrm{E}=123.45\end{array}$ & $\begin{array}{l}\mathrm{O}=1 \\
\mathrm{E}=5.55\end{array}$ & $3 \times 10^{-4}$ \\
\hline $66^{* *}$ & Albian & $\begin{aligned} \mathrm{O} & =62 \\
\mathrm{E} & =47.32\end{aligned}$ & $\begin{aligned} \mathrm{O} & =4 \\
\mathrm{E} & =18.68\end{aligned}$ & $\begin{array}{l}\mathrm{O}=166 \\
\mathrm{E}=180.68\end{array}$ & $\begin{aligned} \mathrm{O} & =86 \\
\mathrm{E} & =71.32\end{aligned}$ & $<1 \times 10^{-5}$ \\
\hline 67 & Cenomanian & $\begin{array}{l}\mathrm{O}=23 \\
\mathrm{E}=21.82\end{array}$ & $\begin{array}{l}\mathrm{O}=1 \\
\mathrm{E}=2.18\end{array}$ & $\begin{array}{l}\mathrm{O}=37 \\
\mathrm{E}=38.18\end{array}$ & $\begin{array}{l}\mathrm{O}=5 \\
\mathrm{E}=3.82\end{array}$ & 0.2943 \\
\hline 68 & Turonian & $\begin{aligned} \mathrm{O} & =11 \\
\mathrm{E} & =10.72\end{aligned}$ & $\begin{array}{l}\mathrm{O}=0 \\
\mathrm{E}=0.28\end{array}$ & $\begin{array}{l}\mathrm{O}=65 \\
\mathrm{E}=65.28\end{array}$ & $\begin{array}{l}\mathrm{O}=2 \\
\mathrm{E}=1.72\end{array}$ & 0.5633 \\
\hline 69 & Coniacian & $\begin{aligned} \mathrm{O} & =5 \\
\mathrm{E} & =4.86\end{aligned}$ & $\begin{array}{l}\mathrm{O}=0 \\
\mathrm{E}=0.14\end{array}$ & $\begin{aligned} \mathrm{O} & =64 \\
\mathrm{E} & =64.14\end{aligned}$ & $\begin{array}{l}\mathrm{O}=2 \\
\mathrm{E}=1.86\end{array}$ & 0.6938 \\
\hline 70 & Santonian & $\begin{array}{l}\mathrm{O}=3 \\
\mathrm{E}=3.00\end{array}$ & $\begin{array}{l}\mathrm{O}=0 \\
\mathrm{E}=0\end{array}$ & $\begin{array}{l}\mathrm{O}=46 \\
\mathrm{E}=46.00\end{array}$ & $\begin{array}{l}\mathrm{O}=0 \\
\mathrm{E}=0\end{array}$ & 1 \\
\hline 71 & Campanian & $\begin{array}{l}\mathrm{O}=7 \\
\mathrm{E}=6.50\end{array}$ & $\begin{array}{l}\mathrm{O}=0 \\
\mathrm{E}=0.50\end{array}$ & $\begin{array}{l}\mathrm{O}=137 \\
\mathrm{E}=137.50\end{array}$ & $\begin{array}{l}\mathrm{O}=11 \\
\mathrm{E}=10.50\end{array}$ & 0.4527 \\
\hline 72 & Maastrichtian & $\begin{array}{l}\mathrm{O}=5 \\
\mathrm{E}=7.93\end{array}$ & $\begin{array}{l}\mathrm{O}=5 \\
\mathrm{E}=2.07\end{array}$ & $\begin{array}{l}\mathrm{O}=202 \\
\mathrm{E}=199.07\end{array}$ & $\begin{aligned} \mathrm{O} & =49 \\
\mathrm{E} & =51.93\end{aligned}$ & 0.0198 \\
\hline
\end{tabular}

version of the data set still fails the chi-square test $(p=0.23$, Table 10$)$.

Analyses 123-128 (Table 10) exclude the U.S. trackway data. Four of these analyses $(124,125,127$, and 128) fail the chi-square test. The trackway data alone (Analyses 123 and 126) pass the chi-square tests $\left(p=1 \times 10^{-5}\right)$ and still support the associations between wide-gauge trackways and inland environments and narrow-gauge trackways and coastal environments.

Finally, Analyses 129-134 (Table 10) explore the consequences of excluding both the Korean and U.S. trackway data (56\% of the tracksite data set). Analyses 129 and 131 (based on number of individuals) both pass the chi-square test $\left(p<6 \times 10^{-4}\right.$, Table 10), with the former supporting the positive association between titanosaurs/wide-gauge trackways and inland environments and nontitanosaurs/narrow-gauge trackways and coastal environments, but the latter reversing the polarity of this association. Analysis 131 is not surprising, however, because several other analyses based on the titanosauriform/non-titanosauriform categories produce similar results (e.g., Analysis 8, Table 2). The three remaining locality-based analyses (nos. 132-134) all fail the chi-square tests (Table 10).

\section{Discussion}

Environmental Associations among Sauropods

In this section we discuss the taxonomic level at which we believe the environmental associations hold true, and the polarity of 
TABle 7. Results of Analyses 73-86 (sauropod body fossil localities) per European stage. Only stages where both titanosaurs and non-titanosaurs are known are shown. See Table 2 for explanation of abbreviations and symbols.

\begin{tabular}{|c|c|c|c|c|c|c|}
\hline \multirow[b]{2}{*}{ Analysis no. } & \multirow[b]{2}{*}{ Stage } & \multicolumn{2}{|c|}{ Non-titanosaurs } & \multicolumn{2}{|c|}{ Titanosaurs } & \multirow[b]{2}{*}{$p$-value } \\
\hline & & Inland & Coastal & Inland & Coastal & \\
\hline $73^{* *}$ & Kimmeridgian & $\begin{array}{l}\mathrm{O}=235 \\
\mathrm{E}=233.10\end{array}$ & $\begin{array}{l}\mathrm{O}=23 \\
\mathrm{E}=24.90\end{array}$ & $\begin{aligned} \mathrm{O} & =0 \\
\mathrm{E} & =4.52\end{aligned}$ & $\begin{aligned} \mathrm{O} & =5 \\
\mathrm{E} & =0.48\end{aligned}$ & $<1 \times 10^{-5}$ \\
\hline $74^{* *}$ & Tithonian & $\begin{array}{l}\mathrm{O}=225 \\
\mathrm{E}=222.57\end{array}$ & $\begin{aligned} \mathrm{O} & =50 \\
\mathrm{E} & =52.43\end{aligned}$ & $\begin{array}{l}\mathrm{O}=0 \\
\mathrm{E}=5.67\end{array}$ & $\begin{array}{l}\mathrm{O}=7 \\
\mathrm{E}=1.33\end{array}$ & $<1 \times 10^{-5}$ \\
\hline 75 & Berriasian & $\begin{array}{l}\mathrm{O}=17 \\
\mathrm{E}=17.00\end{array}$ & $\begin{aligned} \mathrm{O} & =0 \\
\mathrm{E} & =0\end{aligned}$ & $\begin{array}{l}\mathrm{O}=6 \\
\mathrm{E}=6.00\end{array}$ & $\begin{array}{l}\mathrm{O}=0 \\
\mathrm{E}=0\end{array}$ & 1 \\
\hline 76 & Valanginian & $\begin{aligned} \mathrm{O} & =10 \\
\mathrm{E} & =10.00\end{aligned}$ & $\begin{array}{l}\mathrm{O}=0 \\
\mathrm{E}=0\end{array}$ & $\begin{aligned} \mathrm{O} & =15 \\
\mathrm{E} & =15.00\end{aligned}$ & $\begin{aligned} \mathrm{O} & =0 \\
\mathrm{E} & =0\end{aligned}$ & 1 \\
\hline 77 & Hauterivian & $\begin{array}{l}\mathrm{O}=22 \\
\mathrm{E}=24.70\end{array}$ & $\begin{array}{l}\mathrm{O}=4 \\
\mathrm{E}=1.30\end{array}$ & $\begin{array}{l}\mathrm{O}=16 \\
\mathrm{E}=15.2\end{array}$ & $\begin{array}{l}\mathrm{O}=0 \\
\mathrm{E}=0.8\end{array}$ & 0.0094 \\
\hline 78 & Barremian & $\begin{aligned} \mathrm{O} & =34 \\
\mathrm{E} & =36.8\end{aligned}$ & $\begin{array}{l}\mathrm{O}=12 \\
\mathrm{E}=9.20\end{array}$ & $\begin{array}{l}\mathrm{O}=15 \\
\mathrm{E}=12.00\end{array}$ & $\begin{array}{l}\mathrm{O}=0 \\
\mathrm{E}=3.00\end{array}$ & 0.0282 \\
\hline 79 & Aptian & $\begin{array}{l}\mathrm{O}=32 \\
\mathrm{E}=34.07\end{array}$ & $\begin{array}{l}O=6 \\
E=3.93\end{array}$ & $\begin{array}{l}\mathrm{O}=22 \\
\mathrm{E}=19.72\end{array}$ & $\begin{array}{l}\mathrm{O}=0 \\
\mathrm{E}=2.28\end{array}$ & 0.0525 \\
\hline 80 & Albian & $\begin{array}{l}\mathrm{O}=42 \\
\mathrm{E}=43.17\end{array}$ & $\begin{array}{l}\mathrm{O}=4 \\
\mathrm{E}=2.83\end{array}$ & $\begin{array}{l}\mathrm{O}=23 \\
\mathrm{E}=21.58\end{array}$ & $\begin{array}{l}\mathrm{O}=0 \\
\mathrm{E}=1.42\end{array}$ & 0.1552 \\
\hline 81 & Cenomanian & $\begin{aligned} \mathrm{O} & =19 \\
\mathrm{E} & =19.05\end{aligned}$ & $\begin{array}{l}\mathrm{O}=1 \\
\mathrm{E}=0.95\end{array}$ & $\begin{array}{l}\mathrm{O}=26 \\
\mathrm{E}=25.71\end{array}$ & $\begin{array}{l}\mathrm{O}=1 \\
\mathrm{E}=1.29\end{array}$ & 0.7153 \\
\hline 82 & Turonian & $\begin{aligned} \mathrm{O} & =10 \\
\mathrm{E} & =10.00\end{aligned}$ & $\begin{array}{l}\mathrm{O}=0 \\
\mathrm{E}=0\end{array}$ & $\begin{aligned} \mathrm{O} & =41 \\
\mathrm{E} & =41.00\end{aligned}$ & $\begin{array}{l}\mathrm{O}=0 \\
\mathrm{E}=0\end{array}$ & 1 \\
\hline 83 & Coniacian & $\begin{aligned} \mathrm{O} & =4 \\
\mathrm{E} & =4.00\end{aligned}$ & $\begin{array}{l}\mathrm{O}=0 \\
\mathrm{E}=0\end{array}$ & $\begin{array}{l}\mathrm{O}=40 \\
\mathrm{E}=40.00\end{array}$ & $\begin{array}{l}\mathrm{O}=0 \\
\mathrm{E}=0\end{array}$ & 1 \\
\hline 84 & Santonian & $\begin{array}{l}\mathrm{O}=2 \\
\mathrm{E}=2.00\end{array}$ & $\begin{array}{l}\mathrm{O}=0 \\
\mathrm{E}=0\end{array}$ & $\begin{array}{l}\mathrm{O}=40 \\
\mathrm{E}=40.00\end{array}$ & $\begin{array}{l}\mathrm{O}=0 \\
\mathrm{E}=0\end{array}$ & 1 \\
\hline 85 & Campanian & $\begin{array}{l}\mathrm{O}=3 \\
\mathrm{E}=2.93\end{array}$ & $\begin{array}{l}\mathrm{O}=0 \\
\mathrm{E}=0.07\end{array}$ & $\begin{array}{l}\mathrm{O}=81 \\
\mathrm{E}=80.98\end{array}$ & $\begin{array}{l}\mathrm{O}=2 \\
\mathrm{E}=2.02\end{array}$ & 0.7592 \\
\hline $86^{*}$ & Maastrichtian & $\begin{array}{l}\mathrm{O}=4 \\
\mathrm{E}=5.90\end{array}$ & $\begin{array}{l}\mathrm{O}=2 \\
\mathrm{E}=0.10\end{array}$ & $\begin{array}{l}\mathrm{O}=113 \\
\mathrm{E}=111.08\end{array}$ & $\begin{array}{l}\mathrm{O}=0 \\
\mathrm{E}=1.92\end{array}$ & $<1 \times 10^{-5}$ \\
\hline
\end{tabular}

TABLE 8. Results of Analyses 87-98 (sauropod tracksite localities) per European stage. Only stages where both titanosaurs and non-titanosaurs are known are shown. See Table 2 for explanation of abbreviations and symbols.

\begin{tabular}{|c|c|c|c|c|c|c|}
\hline \multirow[b]{2}{*}{ Analysis no. } & \multirow[b]{2}{*}{ Stage } & \multicolumn{2}{|c|}{ Narrow-gauge } & \multicolumn{2}{|c|}{ Wide-gauge } & \multirow[b]{2}{*}{$p$-value } \\
\hline & & Inland & Coastal & Inland & Coastal & \\
\hline 87 & Bajocian & $\begin{array}{l}\mathrm{O}=1 \\
\mathrm{E}=0.67\end{array}$ & $\begin{array}{l}\mathrm{O}=1 \\
\mathrm{E}=1.33\end{array}$ & $\begin{array}{l}\mathrm{O}=0 \\
\mathrm{E}=0.33\end{array}$ & $\begin{array}{l}\mathrm{O}=1 \\
\mathrm{E}=0.67\end{array}$ & 0.3913 \\
\hline 88 & Bathonian & $\begin{array}{l}\mathrm{O}=6 \\
\mathrm{E}=5.57\end{array}$ & $\begin{array}{l}\mathrm{O}=7 \\
\mathrm{E}=7.43\end{array}$ & $\begin{array}{l}\mathrm{O}=0 \\
\mathrm{E}=0.86\end{array}$ & $\begin{array}{l}\mathrm{O}=2 \\
\mathrm{E}=1.14\end{array}$ & 0.2106 \\
\hline 89 & Callovian & $\begin{array}{l}\mathrm{O}=1 \\
\mathrm{E}=1.33\end{array}$ & $\begin{array}{l}\mathrm{O}=1 \\
\mathrm{E}=0.67\end{array}$ & $\begin{array}{l}\mathrm{O}=1 \\
\mathrm{E}=0.67\end{array}$ & $\begin{array}{l}\mathrm{O}=0 \\
\mathrm{E}=0.33\end{array}$ & 0.3871 \\
\hline 90 & Kimmeridgian & $\begin{array}{l}\mathrm{O}=4 \\
\mathrm{E}=4.20\end{array}$ & $\begin{array}{l}\mathrm{O}=8 \\
\mathrm{E}=7.80\end{array}$ & $\begin{array}{l}\mathrm{O}=3 \\
\mathrm{E}=2.80\end{array}$ & $\begin{array}{l}\mathrm{O}=5 \\
\mathrm{E}=5.20\end{array}$ & 0.8495 \\
\hline 91 & Tithonian & $\begin{array}{l}\mathrm{O}=3 \\
\mathrm{E}=4.00\end{array}$ & $\begin{array}{l}\mathrm{O}=9 \\
\mathrm{E}=8.00\end{array}$ & $\begin{array}{l}\mathrm{O}=6 \\
\mathrm{E}=5.00\end{array}$ & $\begin{array}{l}\mathrm{O}=9 \\
\mathrm{E}=10.00\end{array}$ & 0.4113 \\
\hline 92 & Berriasian & $\begin{aligned} \mathrm{O} & =0 \\
\mathrm{E} & =0.70\end{aligned}$ & $\begin{array}{l}\mathrm{O}=1 \\
\mathrm{E}=0.30\end{array}$ & $\begin{array}{l}\mathrm{O}=7 \\
\mathrm{E}=6.30\end{array}$ & $\begin{array}{l}\mathrm{O}=2 \\
\mathrm{E}=2.70\end{array}$ & 0.1073 \\
\hline 93 & Valanginian & $\begin{array}{l}\mathrm{O}=0 \\
\mathrm{E}=0.75\end{array}$ & $\begin{array}{l}\mathrm{O}=1 \\
\mathrm{E}=0.25\end{array}$ & $\begin{array}{l}\mathrm{O}=6 \\
\mathrm{E}=5.25\end{array}$ & $\begin{array}{l}\mathrm{O}=1 \\
\mathrm{E}=1.75\end{array}$ & 0.0641 \\
\hline 94 & Hauterivian & $\begin{aligned} \mathrm{O} & =0 \\
\mathrm{E} & =0.75\end{aligned}$ & $\begin{aligned} \mathrm{O} & =1 \\
\mathrm{E} & =0.25\end{aligned}$ & $\begin{aligned} \mathrm{O} & =6 \\
\mathrm{E} & =5.25\end{aligned}$ & $\begin{aligned} \mathrm{O} & =1 \\
\mathrm{E} & =1.75\end{aligned}$ & 0.0641 \\
\hline $95^{*}$ & Barremian & $\begin{aligned} \mathrm{O} & =0 \\
\mathrm{E} & =0.92\end{aligned}$ & $\begin{array}{l}\mathrm{O}=1 \\
\mathrm{E}=0.08\end{array}$ & $\begin{aligned} \mathrm{O} & =11 \\
\mathrm{E} & =10.08\end{aligned}$ & $\begin{aligned} \mathrm{O} & =0 \\
\mathrm{E} & =0.92\end{aligned}$ & $3 \times 10^{-4}$ \\
\hline $96^{*}$ & Aptian & $\begin{array}{l}\mathrm{O}=0 \\
\mathrm{E}=0.96\end{array}$ & $\begin{array}{l}\mathrm{O}=1 \\
\mathrm{E}=0.04\end{array}$ & $\begin{array}{l}\mathrm{O}=49 \\
\mathrm{E}=48.04\end{array}$ & $\begin{array}{l}\mathrm{O}=1 \\
\mathrm{E}=1.96\end{array}$ & $<1 \times 10^{-5}$ \\
\hline 97 & Albian & $\begin{array}{l}\mathrm{O}=1 \\
\mathrm{E}=0.77\end{array}$ & $\begin{array}{l}\mathrm{O}=0 \\
\mathrm{E}=0.23\end{array}$ & $\begin{array}{l}\mathrm{O}=78 \\
\mathrm{E}=78.23\end{array}$ & $\begin{array}{l}\mathrm{O}=24 \\
\mathrm{E}=23.77\end{array}$ & 0.5826 \\
\hline 98 & Campanian & $\begin{array}{l}\mathrm{O}=3 \\
\mathrm{E}=2.14\end{array}$ & $\begin{array}{l}\mathrm{O}=0 \\
\mathrm{E}=0.86\end{array}$ & $\begin{aligned} \mathrm{O} & =2 \\
\mathrm{E} & =2.86\end{aligned}$ & $\begin{array}{l}\mathrm{O}=2 \\
\mathrm{E}=1.14\end{array}$ & 0.1455 \\
\hline
\end{tabular}


TABLE 9. Results of Analyses 99-115 (sauropod tracksite and body fossil localities) per European stage. Only stages where both titanosaurs and non-titanosaurs are known are shown. See Table 2 for explanation of abbreviations and symbols.

\begin{tabular}{|c|c|c|c|c|c|c|}
\hline \multirow[b]{2}{*}{ Analysis no. } & \multirow[b]{2}{*}{ Stage } & \multicolumn{2}{|c|}{$\begin{array}{c}\text { Non-titanosaurs and } \\
\text { narrow-gauge }\end{array}$} & \multicolumn{2}{|c|}{$\begin{array}{c}\text { Titanosaurs and } \\
\text { wide-gauge }\end{array}$} & \multirow[b]{2}{*}{$p$-value } \\
\hline & & Inland & Coastal & Inland & Coastal & \\
\hline 99 & Bajocian & $\begin{aligned} \mathrm{O} & =16 \\
\mathrm{E} & =15.00\end{aligned}$ & $\begin{array}{l}\mathrm{O}=1 \\
\mathrm{E}=2.00\end{array}$ & $\begin{aligned} \mathrm{O} & =0 \\
\mathrm{E} & =0.88\end{aligned}$ & $\begin{aligned} \mathrm{O} & =1 \\
\mathrm{E} & =0.12\end{aligned}$ & 0.0049 \\
\hline 100 & Bathonian & $\begin{array}{l}\mathrm{O}=22 \\
\mathrm{E}=21.00\end{array}$ & $\begin{array}{l}\mathrm{O}=9 \\
\mathrm{E}=10.00\end{array}$ & $\begin{array}{l}\mathrm{O}=0 \\
\mathrm{E}=1.35\end{array}$ & $\begin{array}{l}\mathrm{O}=2 \\
\mathrm{E}=0.65\end{array}$ & 0.0381 \\
\hline 101 & Callovian & $\begin{aligned} O & =21 \\
E & =20.95\end{aligned}$ & $\begin{aligned} \mathrm{O} & =1 \\
\mathrm{E} & =1.05\end{aligned}$ & $\begin{aligned} \mathrm{O} & =1 \\
\mathrm{E} & =0.95\end{aligned}$ & $\begin{aligned} \mathrm{O} & =0 \\
\mathrm{E} & =0.05\end{aligned}$ & 0.8144 \\
\hline $102^{* *}$ & Kimmeridgian & $\begin{array}{l}\mathrm{O}=239 \\
\mathrm{E}=241.86\end{array}$ & $\begin{aligned} \mathrm{O} & =31 \\
\mathrm{E} & =38.14\end{aligned}$ & $\begin{array}{l}\mathrm{O}=3 \\
\mathrm{E}=11.23\end{array}$ & $\begin{array}{l}\mathrm{O}=10 \\
\mathrm{E}=1.77\end{array}$ & $<1 \times 10^{-5}$ \\
\hline $103^{* *}$ & Tithonian & $\begin{array}{l}O=228 \\
E=220.19\end{array}$ & $\begin{array}{l}\mathrm{O}=59 \\
\mathrm{E}=66.81\end{array}$ & $\begin{aligned} \mathrm{O} & =6 \\
\mathrm{E} & =16.88\end{aligned}$ & $\begin{array}{l}\mathrm{O}=16 \\
\mathrm{E}=5.12\end{array}$ & $<1 \times 10^{-5}$ \\
\hline 104 & Berriasian & $\begin{array}{l}\mathrm{O}=17 \\
\mathrm{E}=16.36\end{array}$ & $\begin{aligned} \mathrm{O} & =1 \\
\mathrm{E} & =1.64\end{aligned}$ & $\begin{aligned} \mathrm{O} & =13 \\
\mathrm{E} & =13.64\end{aligned}$ & $\begin{array}{l}\mathrm{O}=2 \\
\mathrm{E}=1.36\end{array}$ & 0.3893 \\
\hline 105 & Valanginian & $\begin{array}{l}\mathrm{O}=10 \\
\mathrm{E}=10.33\end{array}$ & $\begin{array}{l}\mathrm{O}=1 \\
\mathrm{E}=0.67\end{array}$ & $\begin{aligned} \mathrm{O} & =21 \\
\mathrm{E} & =20.67\end{aligned}$ & $\begin{array}{l}\mathrm{O}=1 \\
\mathrm{E}=1.33\end{array}$ & 0.6122 \\
\hline 106 & Hauterivian & $\begin{array}{l}\mathrm{O}=22 \\
\mathrm{E}=24.75\end{array}$ & $\begin{array}{l}\mathrm{O}=5 \\
\mathrm{E}=2.25\end{array}$ & $\begin{array}{l}0=22 \\
\mathrm{E}=21.08\end{array}$ & $\begin{array}{l}\mathrm{O}=1 \\
\mathrm{E}=1.92\end{array}$ & 0.0416 \\
\hline 107 & Barremian & $\begin{aligned} \mathrm{O} & =34 \\
\mathrm{E} & =38.51\end{aligned}$ & $\begin{array}{l}\mathrm{O}=13 \\
\mathrm{E}=8.49\end{array}$ & $\begin{aligned} \mathrm{O} & =26 \\
\mathrm{E} & =21.31\end{aligned}$ & $\begin{array}{l}\mathrm{O}=0 \\
\mathrm{E}=4.69\end{array}$ & 0.0033 \\
\hline 108 & Aptian & $\begin{aligned} \mathrm{O} & =32 \\
\mathrm{E} & =36.14\end{aligned}$ & $\begin{array}{l}\mathrm{O}=7 \\
\mathrm{E}=2.86\end{array}$ & $\begin{array}{l}\mathrm{O}=71 \\
\mathrm{E}=66.72\end{array}$ & $\begin{aligned} \mathrm{O} & =1 \\
\mathrm{E} & =5.28\end{aligned}$ & 0.0014 \\
\hline 109 & Albian & $\begin{array}{l}\mathrm{O}=43 \\
\mathrm{E}=39.17\end{array}$ & $\begin{array}{l}\mathrm{O}=4 \\
\mathrm{E}=7.83\end{array}$ & $\begin{array}{l}\mathrm{O}=101 \\
\mathrm{E}=104.17\end{array}$ & $\begin{aligned} \mathrm{O} & =24 \\
\mathrm{E} & =20.83\end{aligned}$ & 0.0932 \\
\hline 110 & Cenomanian & $\begin{array}{l}\mathrm{O}=19 \\
\mathrm{E}=18.64\end{array}$ & $\begin{array}{l}\mathrm{O}=1 \\
\mathrm{E}=1.36\end{array}$ & $\begin{array}{l}\mathrm{O}=27 \\
\mathrm{E}=27.02\end{array}$ & $\begin{aligned} \mathrm{O} & =2 \\
\mathrm{E} & =1.98\end{aligned}$ & 0.7494 \\
\hline 111 & Turonian & $\begin{array}{l}\mathrm{O}=10 \\
\mathrm{E}=9.81\end{array}$ & $\begin{array}{l}\mathrm{O}=0 \\
\mathrm{E}=0.19\end{array}$ & $\begin{aligned} \mathrm{O} & =45 \\
\mathrm{E} & =45.15\end{aligned}$ & $\begin{array}{l}\mathrm{O}=1 \\
\mathrm{E}=0.85\end{array}$ & 0.6383 \\
\hline 112 & Coniacian & $\begin{array}{l}\mathrm{O}=4 \\
\mathrm{E}=3.91\end{array}$ & $\begin{array}{l}\mathrm{O}=0 \\
\mathrm{E}=0.09\end{array}$ & $\begin{array}{l}\mathrm{O}=44 \\
\mathrm{E}=44.04\end{array}$ & $\begin{array}{l}\mathrm{O}=1 \\
\mathrm{E}=0.96\end{array}$ & 0.7655 \\
\hline 113 & Santonian & $\begin{array}{l}\mathrm{O}=2 \\
\mathrm{E}=2.00\end{array}$ & $\begin{array}{l}\mathrm{O}=0 \\
\mathrm{E}=0\end{array}$ & $\begin{aligned} \mathrm{O} & =40 \\
\mathrm{E} & =40.00\end{aligned}$ & $\begin{array}{l}\mathrm{O}=0 \\
\mathrm{E}=0\end{array}$ & 1 \\
\hline 114 & Campanian & $\begin{array}{l}\mathrm{O}=6 \\
\mathrm{E}=5.73\end{array}$ & $\begin{array}{l}\mathrm{O}=0 \\
\mathrm{E}=0.27\end{array}$ & $\begin{array}{l}\mathrm{O}=83 \\
\mathrm{E}=83.05\end{array}$ & $\begin{array}{l}\mathrm{O}=4 \\
\mathrm{E}=3.95\end{array}$ & 0.5941 \\
\hline $115^{*}$ & Maastrichtian & $\begin{array}{l}\mathrm{O}=4 \\
\mathrm{E}=5.76\end{array}$ & $\begin{array}{l}\mathrm{O}=2 \\
\mathrm{E}=0.24\end{array}$ & $\begin{array}{l}\mathrm{O}=115 \\
\mathrm{E}=114.16\end{array}$ & $\begin{array}{l}\mathrm{O}=4 \\
\mathrm{E}=4.84\end{array}$ & $2 \times 10^{-4}$ \\
\hline
\end{tabular}

these associations. Whether these associations represent genuine habitat preferences or are merely sampling artifacts is also addressed, with possible explanations provided for each hypothesis.

Taxonomic Level and Association Polarity.All of the statistically significant results were obtained in analyses involving titanosaurs/non-titanosaurs, titanosauriforms/nontitanosauriforms, and/or wide-gauge/narrowgauge trackways, whereas none of the results involving Macronaria/non-Macronaria were statistically significant. These results indicate that Butler et al.'s (2007) and Butler and Barrett's (2008) suggestion that macronarians are positively associated with inland habitats is probably incorrect (N.B. these authors were also skeptical about the significance of their macronarian result, suggesting that it might have been generated by a taphonomic artifact). By limiting their data set to Cretaceous forms, Butler and colleagues effectively restricted composition of this macronarian group largely to titanosauriform taxa because virtually all non-titanosauriform macronarians are Jurassic in age (e.g., Camarasaurus). Had they instead considered all sauropods, the positive association between macronarians and inland environments would have been much weaker or perhaps nonexistent. The reason our analyses at the Macronaria/non-Macronaria level, even when restricted to Cretaceous forms, failed to support an environmental association is that we used a larger data set: when marine 
TABle 10. Results of Analyses 116-134 ('Sensitivity analyses). See Table 2 for explanation of abbreviations and symbols.

\begin{tabular}{|c|c|c|c|c|}
\hline Analysis no. & Comparison & Inland & Coastal & $p$-value \\
\hline \multirow[t]{5}{*}{$116^{*}$} & Trackway individuals minus Korean data: $\mathrm{T}=519$ & $\mathrm{~T}=122$ & $\mathrm{~T}=397$ & $<1 \times 10^{-5}$ \\
\hline & Narrow-gauge: $\mathrm{T}=183$ & $\mathrm{O}=26$ & $\mathrm{O}=157$ & \\
\hline & & $E=43.02$ & $E=139.98$ & \\
\hline & Wide-gauge $\mathrm{T}=336$ & $\mathrm{O}=96$ & $\mathrm{O}=240$ & \\
\hline & & $\mathrm{E}=78.98$ & $E=257.02$ & \\
\hline \multirow[t]{5}{*}{$117^{* *}$} & $\begin{array}{l}\text { Body fossil and trackway individuals minus Korean } \\
\text { data: } \mathrm{T}=1879\end{array}$ & $\mathrm{~T}=1348$ & $\mathrm{~T}=531$ & $<1 \times 10^{-5}$ \\
\hline & Non-titanosaurs and narrow-gauge: $\mathrm{T}=1170$ & $\mathrm{O}=890$ & $\mathrm{O}=280$ & \\
\hline & & $E=839.36$ & $E=330.64$ & \\
\hline & Titanosaurs and wide-gauge: $\mathrm{T}=709$ & $\mathrm{O}=458$ & $\mathrm{O}=251$ & \\
\hline & & $E=508.64$ & $E=200.36$ & \\
\hline \multirow[t]{5}{*}{ 118\#\# } & $\begin{array}{l}\text { Body fossil and trackway individuals minus Korean } \\
\text { data: } T=1879\end{array}$ & $\mathrm{~T}=1348$ & $\mathrm{~T}=531$ & $<1 \times 10^{-5}$ \\
\hline & Non-titanosauriforms and narrow-gauge: $\mathrm{T}=1002$ & $\mathrm{O}=771$ & $\mathrm{O}=231$ & \\
\hline & & $\mathrm{E}=718.84$ & $E=283.16$ & \\
\hline & Titanosauriforms and wide-gauge: $\mathrm{T}=877$ & $\mathrm{O}=577$ & $\mathrm{O}=300$ & \\
\hline & & $E=629.16$ & $E=247.84$ & \\
\hline \multirow[t]{5}{*}{119} & Trackway localities minus Korean data: $\mathrm{T}=117$ & $\mathrm{~T}=43$ & $\mathrm{~T}=74$ & 0.2432 \\
\hline & Narrow-gauge: $\mathrm{T}=38$ & $\mathrm{O}=11$ & $\mathrm{O}=27$ & \\
\hline & & $\mathrm{E}=13.97$ & $\mathrm{E}=24.03$ & \\
\hline & Wide-gauge: $\mathrm{T}=80$ & $\mathrm{O}=32$ & $\mathrm{O}=48$ & \\
\hline & & $E=29.40$ & $E=50.60$ & \\
\hline \multirow[t]{5}{*}{120} & $\begin{array}{l}\text { Body fossil and trackway localities minus Korean } \\
\text { data: } T=823\end{array}$ & $\mathrm{~T}=667$ & $\mathrm{~T}=156$ & 0.5896 \\
\hline & Non-titanosaurs and narrow-gauge: $\mathrm{T}=517$ & $\mathrm{O}=415$ & $\mathrm{O}=102$ & \\
\hline & & $E=419.00$ & $\mathrm{E}=98.00$ & \\
\hline & Titanosaurs and wide-gauge: $\mathrm{T}=317$ & $\mathrm{O}=259$ & $\mathrm{O}=58$ & \\
\hline & & $E=256.91$ & $E=60.09$ & \\
\hline \multirow[t]{5}{*}{121} & $\begin{array}{l}\text { Body fossil and trackway localities minus Korean } \\
\text { data: } T=823\end{array}$ & $\mathrm{~T}=667$ & $\mathrm{~T}=156$ & 0.0513 \\
\hline & Non-titanosauriforms and narrow-gauge: $\mathrm{T}=396$ & $\mathrm{O}=334$ & $\mathrm{O}=74$ & \\
\hline & & $E=320.94$ & $\mathrm{E}=75.06$ & \\
\hline & Titanosauriforms and wide-gauge: $\mathrm{T}=438$ & $\mathrm{O}=341$ & $\mathrm{O}=98$ & \\
\hline & & $E=354.98$ & $\mathrm{E}=83.02$ & \\
\hline \multirow[t]{5}{*}{122} & $\begin{array}{l}\text { Trackway localities with Korea reduced to the } 6 \text { main } \\
\text { localities: } \mathrm{T}=123\end{array}$ & $\mathrm{~T}=49$ & $\mathrm{~T}=74$ & 0.23 \\
\hline & Narrow-gauge: $\mathrm{T}=39$ & $\mathrm{O}=12$ & $\mathrm{O}=27$ & \\
\hline & & $E=15.54$ & $E=23.46$ & \\
\hline & Wide-gauge: $\mathrm{T}=85$ & $\mathrm{O}=37$ & $\mathrm{O}=48$ & \\
\hline & & $E=33.86$ & $\mathrm{E}=51.14$ & \\
\hline \multirow[t]{5}{*}{$123^{*}$} & Trackway individuals minus USA data: $\mathrm{T}=502$ & $\mathrm{~T}=222$ & $\mathrm{~T}=280$ & $<1 \times 10^{-5}$ \\
\hline & Narrow-gauge: $\mathrm{T}=141$ & $\mathrm{O}=27$ & $\mathrm{O}=114$ & \\
\hline & & $E=62.35$ & $E=78.65$ & \\
\hline & Wide-gauge: $\mathrm{T}=361$ & $\mathrm{O}=195$ & $\mathrm{O}=166$ & \\
\hline & & $E=159.65$ & $E=201.35$ & \\
\hline \multirow[t]{5}{*}{124} & $\begin{array}{l}\text { Body fossil and trackway individuals minus USA } \\
\text { data: } \mathrm{T}=1862\end{array}$ & $\mathrm{~T}=1448$ & $\mathrm{~T}=414$ & 0.1143 \\
\hline & Non-titanosaurs and narrow-gauge: $\mathrm{T}=1128$ & $\mathrm{O}=891$ & $\mathrm{O}=237$ & \\
\hline & & $\mathrm{E}=877.20$ & $E=250.80$ & \\
\hline & Titanosaurs and wide-gauge: $\mathrm{T}=734$ & $\mathrm{O}=557$ & $\mathrm{O}=177$ & \\
\hline & & $E=570.80$ & $E=163.20$ & \\
\hline \multirow[t]{5}{*}{125} & $\begin{array}{l}\text { Body fossil and trackway individuals minus USA } \\
\text { data: } \mathrm{T}=1862\end{array}$ & $\mathrm{~T}=1448$ & $\mathrm{~T}=414$ & 0.0044 \\
\hline & Non-titanosauriforms and narrow-gauge: $\mathrm{T}=960$ & $\mathrm{O}=772$ & $\mathrm{O}=188$ & \\
\hline & & $E=746.55$ & $E=213.45$ & \\
\hline & Titanosauriforms and wide-gauge: $\mathrm{T}=902$ & $\mathrm{O}=676$ & $\mathrm{O}=226$ & \\
\hline & & $\mathrm{E}=701.45$ & $E=200.55$ & \\
\hline \multirow[t]{5}{*}{$126^{*}$} & Trackway localities minus USA data: $\mathrm{T}=157$ & $\mathrm{~T}=107$ & $\mathrm{~T}=50$ & $<1 \times 10^{-5}$ \\
\hline & Narrow-gauge: $\mathrm{T}=34$ & $\mathrm{O}=11$ & $\mathrm{O}=23$ & \\
\hline & & $\mathrm{E}=23.17$ & $\mathrm{E}=10.83$ & \\
\hline & Wide-gauge: $\mathrm{T}=124$ & $\mathrm{O}=96$ & $\mathrm{O}=28$ & \\
\hline & & $\mathrm{E}=84.51$ & $\mathrm{E}=39.49$ & \\
\hline
\end{tabular}


TABLE 10. Continued.

\begin{tabular}{|c|c|c|c|c|}
\hline Analysis no. & Comparison & Inland & Coastal & $p$-value \\
\hline \multirow[t]{5}{*}{127} & $\begin{array}{l}\text { Body fossil and trackway localities minus USA data: } \\
\mathrm{T}=863\end{array}$ & $\mathrm{~T}=731$ & $\mathrm{~T}=132$ & $5 \times 10^{-4}$ \\
\hline & Non-titanosaurs and narrow-gauge: $\mathrm{T}=513$ & $\mathrm{O}=415$ & $\mathrm{O}=98$ & \\
\hline & & $E=434.53$ & $\mathrm{E}=78.47$ & \\
\hline & Titanosaurs and wide-gauge: $\mathrm{T}=361$ & $\mathrm{O}=323$ & $\mathrm{O}=38$ & \\
\hline & & $E=305.78$ & $\mathrm{E}=55.22$ & \\
\hline \multirow[t]{4}{*}{128} & $\begin{array}{l}\text { Body fossil and trackway localities minus USA data: } \\
\mathrm{T}=863\end{array}$ & $\mathrm{~T}=731$ & $\mathrm{~T}=132$ & 0.2121 \\
\hline & Non-titanosauriforms and narrow-gauge: $\mathrm{T}=404$ & $334(342.21)$ & $70(61.79)$ & \\
\hline & Titanosauriforms and wide-gauge: $\mathrm{T}=483$ & $\mathrm{O}=405$ & $\mathrm{O}=78$ & \\
\hline & & $\mathrm{E}=409.12$ & $\mathrm{E}=73.88$ & \\
\hline \multirow[t]{5}{*}{$129^{*}$} & $\begin{array}{l}\text { Trackway individuals minus Korean and USA data: } \\
\mathrm{T}=388\end{array}$ & $\mathrm{~T}=108$ & $\mathrm{~T}=280$ & $3 \times 10^{-4}$ \\
\hline & Narrow-gauge: $\mathrm{T}=137$ & $\mathrm{O}=23$ & $\mathrm{O}=114$ & \\
\hline & & $\mathrm{E}=38.13$ & $\mathrm{E}=98.87$ & \\
\hline & Wide-gauge: $\mathrm{T}=251$ & $\mathrm{O}=85$ & $\mathrm{O}=166$ & \\
\hline & & $E=69.87$ & $\mathrm{E}=181.13$ & \\
\hline \multirow[t]{5}{*}{130} & $\begin{array}{l}\text { Body fossil and trackway individuals minus Korean } \\
\text { and USA data: } T=1748\end{array}$ & $\mathrm{~T}=1334$ & $\mathrm{~T}=414$ & $6 \times 10^{-4}$ \\
\hline & Non-titanosaurs and narrow-gauge: $\mathrm{T}=1124$ & $\mathrm{O}=887$ & $\mathrm{O}=237$ & \\
\hline & & $\mathrm{E}=857.79$ & $\mathrm{E}=266.21$ & \\
\hline & Titanosaurs and wide-gauge: $\mathrm{T}=624$ & $\mathrm{O}=447$ & $\mathrm{O}=177$ & \\
\hline & & $E=476.21$ & $\mathrm{E}=147.79$ & \\
\hline \multirow[t]{5}{*}{ 131\#\# } & $\begin{array}{l}\text { Body fossil and trackway individuals minus Korean } \\
\text { and USA data: } \mathrm{T}=1748\end{array}$ & $\mathrm{~T}=1334$ & $\mathrm{~T}=414$ & $1 \times 10^{-5}$ \\
\hline & Non-titanosauriforms and narrow-gauge: $\mathrm{T}=956$ & $\mathrm{O}=768$ & $\mathrm{O}=188$ & \\
\hline & & $E=729.58$ & $E=226.42$ & \\
\hline & Titanosauriforms and wide-gauge: $\mathrm{T}=792$ & $\mathrm{O}=566$ & $\mathrm{O}=226$ & \\
\hline & & $E=604.42$ & $\mathrm{E}=187.58$ & \\
\hline \multirow[t]{5}{*}{132} & $\begin{array}{l}\text { Trackway localities minus USA and Korean data: } \mathrm{T}= \\
84\end{array}$ & $\mathrm{~T}=34$ & $\mathrm{~T}=50$ & 0.0788 \\
\hline & Narrow-gauge: $\mathrm{T}=31$ & $\mathrm{O}=8$ & $\mathrm{O}=23$ & \\
\hline & & $\mathrm{E}=12.55$ & $\mathrm{E}=18.45$ & \\
\hline & Wide-gauge: $\mathrm{T}=54$ & $\mathrm{O}=26$ & $\mathrm{O}=28$ & \\
\hline & & $E=21.86$ & $\mathrm{E}=32.14$ & \\
\hline \multirow[t]{5}{*}{133} & $\begin{array}{l}\text { Body fossil and trackway localities minus Korean and } \\
\text { USA data: } T=790\end{array}$ & $\mathrm{~T}=658$ & $\mathrm{~T}=132$ & 0.0241 \\
\hline & Non-titanosaurs and narrow-gauge: $\mathrm{T}=510$ & $\mathrm{O}=412$ & $\mathrm{O}=98$ & \\
\hline & & $E=424.78$ & $\mathrm{E}=85.22$ & \\
\hline & Titanosaurs and wide-gauge: $\mathrm{T}=291$ & $\mathrm{O}=253$ & $\mathrm{O}=38$ & \\
\hline & & $E=242.38$ & $E=48.62$ & \\
\hline \multirow[t]{5}{*}{134} & $\begin{array}{l}\text { Body fossil and trackway localities minus Korean and } \\
\text { USA data: } T=790\end{array}$ & $\mathrm{~T}=658$ & $\mathrm{~T}=132$ & 0.2102 \\
\hline & Non-titanosauriforms and narrow-gauge: $\mathrm{T}=401$ & $\mathrm{O}=331$ & $\mathrm{O}=70$ & \\
\hline & & $\mathrm{E}=334.00$ & $\mathrm{E}=67.00$ & \\
\hline & Titanosauriforms and wide-gauge: $\mathrm{T}=413$ & $\mathrm{O}=335$ & $\mathrm{O}=78$ & \\
\hline & & $\mathrm{E}=344.00$ & $\mathrm{E}=69.00$ & \\
\hline
\end{tabular}

deposits and indeterminate sauropod specimens are excluded, the Butler and Barrett (2008) data set comprises 177 Cretaceous sauropod-bearing localities, whereas the equivalent figure for our data set is 475 . This increase reflects additions since Butler collected his data in 2006 as well as personal observations of museum collections that added substantially to our data set.

Determination of the environmental associations of Titanosauriformes and Titano- sauria is bound up with the issue of polarity (i.e., whether the positive association is with inland or coastal habitats). Of the 41 analyses that produced statistically significant results, 24 support a positive association between titanosaurs, titanosauriforms, and/or widegauge trackways and inland environments, and 17 support the opposite (negative) association. For ease of discussion, we will term these conflicting patterns "titanosaurs prefer inland" and "titanosaurs prefer coastal." 
Detailed examination of these results reveals some important and interesting patterns that help to resolve this conflict.

The results of Analyses 1-10 should carry the greatest weight because they are based on the most data. Analyses 1-5 support the "titanosaurs prefer inland" pattern, and all but Analysis 3 are independent of each other in that they do not share any data in common. Only one (no. 8) of the four analyses based on the total data set supports the "titanosaurs prefer coastal" pattern. One way to reconcile this contradiction is to postulate that basal titanosauriforms were indeed positively associated with coastal environments, and that a less inclusive clade such as Titanosauria subsequently switched its preference to one for inland habitats. This proposal receives support from three separate lines of evidence:

1. Analyses 11 and 12 partition Titanosauriformes into basal forms (non-titanosaurs) and titanosaurs. The results support positive associations between the basal forms and coastal environments and between the more derived titanosaurs and inland environments.

2. Of the 101 time-sliced analyses (Tables 39), 14 support the "titanosaurs prefer inland" pattern and 12 support the opposite pattern. However, 11 of the 12 contradictory analyses were generated by Middle and Late Jurassic time slices, whereas all 14 of the results supporting the inland preference were generated by Cretaceous time slices. Many of these time-sliced analyses are non-independent because they overlap temporally or they are based on common data (e.g., data sets comprising body fossil individuals and data sets comprising these data plus trackway individuals). The 52 stage-level time-sliced analyses in Tables $4,5,7$, and 8 , however, effectively use independent data sets (if we assume, for example, that the number of individuals is independent from the number of localities). Six of these (all Jurassic) support the coastal preference and eight (all Cretaceous) support the preference for inland habitats. This marked temporal division is consistent with the view that the earliest and most basal titanosauriforms and/or titanosaurs occurred more often than expected in coastal habitats, but the more derived Cretaceous forms displayed a preference for inland habitats.

3. The positive association between early and/or basal titanosaurs and coastal habitats could partly be an artifact of taxonomy. There are very few confirmed titanosaur body fossils from the Jurassic: the main evidence is Janenschia robusta from the Kimmeridgian-Tithonian of Tendaguru, Tanzania. Although considered a titanosaur by many workers (Janensch 1929; McIntosh 1990; Jacobs et al. 1993; Upchurch 1995; Wilson and Sereno 1998), both the titanosaurian affinities and congeneric status of the eight individuals currently assigned to this taxon have recently been doubted (Bonaparte et al. 2000). Given that the dinosaur-bearing beds of Tendaguru represent coastal habitats (Aberhan et al. 2002), the incorrect assignment of all or some of the Janenschia individuals to the Titanosauria could have obscured the "titanosaurs prefer inland habitats" pattern, especially for Jurassic time slices.

Habitat Preference or Sampling Artifact?-As discussed earlier, a statistically significant association between an assemblage of taxa and a given environment might not, by itself, provide evidence of a genuine habitat preference. Skewed distributions can arise as a result of other factors, such as long-term trends in taxon diversity and/or abundance combined with long-term trends in the relative sampling rates of the different environmental categories. The observations that titanosaurs appear to be more diverse and abundant during the Cretaceous than during the Jurassic, and that there are approximately three times as many coastal localities (relative to the total number of localities) in the Jurassic compared to the Cretaceous, raise concerns that the skewed distributions of sauropods are artifacts rather than genuine ecological signals. However, when analyses are run at the stage level (i.e., Analyses 30115: Tables 4-9), we find that statistically 
significant environmental associations persist, even though such analyses disrupt the effects of the long-term trend in the relative sampling of inland and coastal environments.

Further support for genuine ecological preferences comes from the observation that only eight localities in our data set have yielded both titanosaurs and non-titanosaurs. Three of these eight localities are Jurassic and coastal whereas the other five are Cretaceous and inland localities (see Supplementary Materials), which is consistent with the hypothesis that the earlier and more basal titanosaurs were more likely to occur in coastal environments than the Cretaceous forms. If titanosaurs and non-titanosaurs had occupied all environments with no discernible preference, many more localities should yield evidence for both types of sauropod coexisting. Although our simple division into inland and coastal categories may be so crude as to partially obscure the precise nature of ecological preferences (see below), the relative rarity of "shared localities" points to genuine ecological partitioning or separation between titanosaurs and non-titanosaurs rather than mere sampling artifacts. We tentatively suggest, therefore, that our results indicate evidence for habitat preferences among sauropod groups, and are not merely artifacts created by trends in the relative sampling of different environments.

Strength of the Habitat Preference.-The raw data (see Supplementary Materials) and the "observed" and "expected" values in Tables 2-10 demonstrate that titanosaur body fossils and/or wide-gauge trackways often occur in coastal habitats, and non-titanosaur body fossils and narrow gauge trackways often occur in inland habitats. For example, consider Analysis 1 (Table 2). In this case (body fossil individuals), there are 864 nontitanosaurs (expected value $=889.75$ ) and 362 titanosaurs (expected value $=336.25$ ) in inland environments. There are fewer nontitanosaurs and more titanosaurs than expected, and this skew is large enough to result in a statistically significant $p$-value. Nevertheless, the observed values indicate that many non-titanosaurs were present in inland environments even though they apparently displayed a preference for coastal environments. Similar observations were made for Cretaceous herbivorous dinosaurian clades by Butler and Barrett (2008). Such results might be interpreted in two ways, which we term the "weak preference hypothesis" and the "strong preference plus noise hypothesis."

The "weak preference hypothesis" suggests that the occurrences of non-titanosaurs and titanosaurs across the inland and coastal environmental categories represent a largely accurate picture of sauropod distributions. Thus, the skewed environmental distributions would reflect a subtle difference between the two groups, such as the relative amounts of time that members of each group spent in each environment, or the relative abundances of each group. If this interpretation is correct, then the difference between titanosaurs and non-titanosaurs should be relatively minor, just large enough to produce a statistically detectable skew in a large data set.

Alternatively, the "strong preference plus noise hypothesis" suggests that the habitat preferences of titanosaurs and non-titanosaurs were significantly different, but that the strength of this signal has been reduced by "noise" in the data set. There are several possible sources of such noise:

- Estimating the number of individuals based on body fossils or trackways is imprecise, and determining the number of localities can also be problematic.

- Evidence of habitat "occupation" may actually be the result of postmortem transport of body fossils. This could be tested by repeating our analyses using a more stringently filtered version of our data set (i.e., by excluding disarticulated or very incomplete specimens), an endeavor that lies outside of the scope of the current study.

- The partitioning of taxa into two categories could introduce noise into an analysis. Suppose, for example, that a genuine difference in habitat preferences existed between titanosaurs and non-titanosaurs. If our analyses are limited to titanosauriforms versus non-titanosauriforms, the results might still be statistically robust 
because the taxonomic contents of Titanosauriformes and Titanosauria are very similar. Nonetheless, Titanosauriformes contains some non-titanosaurs that exhibited a preference for coastal environments, and by including these taxa within the same category as members of Titanosauria, we would weaken the environmental associations signal.

- Taxonomic and phylogenetic errors could obscure or weaken an environmental association signal. Because the phylogenetic relationships of basal titanosaurs and basal titanosauriforms are poorly understood (Upchurch et al. 2004; Curry Rogers 2005), the contents of our titanosaur/nontitanosaur and titanosauriform/non-titanosauriform categories could be inaccurate. Future developments in sauropod phylogenetics might result in some of the taxa we have classified as titanosaurs or titanosauriforms shifting in relative position, producing a strengthening or weakening of the putative environmental associations signal (e.g., see discussion of Janenschia, above).

- Henderson (2006) modeled the position of the center of mass in several sauropods and argued that all large sauropods (over $\sim 12$ tons) would have been constrained to adopt a wide-gauge stance in order to maintain stability during locomotion. This would mean that many large non-titanosaurian taxa, such as Apatosaurus, Camarasaurus, and Turiasaurus, would have produced wide-gauge trackways, potentially introducing errors into all of our "combined evidence" analyses where we have grouped titanosaur body fossils with wide-gauge trackways and non-titanosaur body fossils with narrow-gauge trackways. However, many large-bodied non-titanosaurian sauropods lack most or all of the modifications to the hindlimb which Wilson and Carrano (1999) identified as adaptations for a widegauge stance.

- Our simple division of habitats into inland versus coastal types might partially obscure the true habitat preferences of titanosaurs and non-titanosaurs. If, for example, titanosaurs actually preferred relatively arid conditions, and tended to occupy semi-arid inland environments, then including mesic habitats (e.g., fluvio-lacustrine facies) in the inland category could hide their true habitat preferences. This may explain why, as discussed above, titanosaurs and nontitanosaurs are rarely found at the same localities despite occurring in both habitat categories. Additionally, reports of sauropod skeletons from "fluvial" (inland) environments may obscure the fact that a setting is actually much closer to the coastline (J. A. Wilson personal communication 2009).

We suggest that elements of both the "weak preference hypothesis" and "strong preference plus noise hypothesis" are supported by our data set and analyses. Despite the statistically significant environmental associations of titanosaurs and non-titanosaurs, both types of sauropods probably spent considerable time in both types of habitat. However, one or more sources of noise probably have blurred the habitat preference signal, perhaps making it appear much more subtle than it was.

Nature of the Habitat Preference.-If sauropods did have habitat preferences, then (1) which aspects of the environments were relevant to each group's preference? and (2) Are any of the morphological differences between titanosaurs and non-titanosaurs linked to these habitat preferences? Below we outline two broad hypotheses that might account for the proposed habitat preferences.

The resource exploitation hypothesis suggests that the habitat preference is linked to particular resources in each habitat (such as different types of plant fodder). If this hypothesis is correct, particular plant types should display nonrandom associations with inland and coastal habitats, and titanosaurs and non-titanosaurs should have possessed different feeding mechanisms adapted to exploit these particular resources. Although the patchiness of our sampling of both the sauropod and plant fossil records hampers testing of this idea, several distinctive features of titanosaur skulls and postcrania plausibly can be linked to novel feeding mechanisms (Calvo 1994; Upchurch and 
Barrett 2000; Curry Rogers and Forster 2001; Wilson 2002, 2005b; Upchurch et al. 2004; Barrett and Upchurch 2005). For example, if the wide-gauge stance, increased flexibility of the dorsal vertebral column, short procoelous tails, and anteriorly flaring ilia of titanosaurs are related to a tripod stance (Borsuk-Bialynicka 1977; Powell 1992; Wilson and Carrano 1999), then such a posture may have been used during high-browsing on particular types of plant (though see Henderson 2006).

The locomotion/stance hypothesis proposes a linkage between the wide-gauge stance of titanosaurs (and other anatomical specializations) and some physical (perhaps topographical or substrate-related) aspect of inland environments. The adaptive significance of the wide-gauge stance is still poorly understood, though there can be little doubt that this titanosaurian feature would have had a major effect on many aspects of locomotion and behavior. If, for example, it increased the animal's stability, it might thus have facilitated crossing of uneven or sloping terrain. Wilson and Carrano (1999) noted that the wide-gauge stance is associated with several other anatomical modifications, all of which suggest that titanosaurs had a wider range of motion in the trunk and tail regions and in the fore and hind limbs, which collectively might have enhanced their ability to rear into a tripod stance (Wilson and Carrano 1999) and/ or move more quickly (Apesteguía 2005). Thus, even if inland and coastal habitats possessed approximately the same resources, titanosaurs may have found it easier to exploit these resources in the inland habitats than did non-titanosaurs. This hypothesis could be tested by using the biomechanical approaches proposed by Henderson (2006) and Hutchinson et al. (2007) to model titanosaurs and non-titanosaurs walking and turning at different speeds on a variety of terrains and substrates.

\section{Sauropod Evolutionary History}

Figure 2 illustrates how the diversity of nontitanosaur lineages declined through the Cretaceous, while, at the same time, titanosaurs radiated strongly (see also Barrett and Up- church 2005; Upchurch and Barrett 2005). Why titanosaurs should have been so scarce during the Jurassic and so dominant in the Cretaceous (especially the Late Cretaceous) is not understood, though this pattern probably reflects sampling biases in the fossil record. It is interesting to note, for example, that titanosaurian body fossils are extremely scarce during the Jurassic $(0.01 \%$ of the Jurassic part of our body fossil individual data set), whereas wide-gauge trackways and tracksite localities make up $58 \%$ and $42 \%$ of the Jurassic track data set, respectively. This disparity seems anomalous and may indicate that early titanosaurs occupied environments with low preservation potentials for body fossils, and/or that some of the sauropod taxa known from the Middle and Late Jurassic might be currently unrecognized members of the basal titanosaurian radiation. The decline of non-titanosaurs throughout the Early and early Late Cretaceous is less easily explained as a sampling artifact because we observe a decrease in the abundance and diversity of non-titanosaurian body fossils and narrow-gauge trackways (both are absent from the Coniacian onwards). Table 1 shows that the number of coastal localities producing herbivorous dinosaur material decreases markedly from the Jurassic to the Cretaceous. It seems very improbable that there was a genuine decrease in the number or areal extent of coastal habitats during the Cretaceous; if anything, continental fragmentation during the Cretaceous should have increased the amount of available coastline. If the relative extent of coastal to inland habitats remained the same (or even increased) during the Cretaceous, then the observation that fewer herbivorous dinosaurs were living in coastal environments requires explanation. Sauropods, and perhaps certain ornithischian groups, might have been forced to occupy inland habitats more frequently during the Cretaceous because coastal environments became less hospitable. If nontitanosaurs were less well equipped than titanosaurs to deal with conditions and/or resources in the inland habitats, this might have contributed to their decline in the Early Cretaceous.

In recent years, several authors have remarked on the convergence in feeding and/or 
locomotor systems between diplodocoids (particularly rebbachisaurids) and titanosaurs (Upchurch 1999: pp. 119-120; Curry Rogers and Forster 2004; Apesteguía 2005; Barrett and Upchurch 2005). Rebbachisaurids seem to have diversified during the Early Cretaceous and were the last non-titanosaurian group of sauropods to go extinct. Although these observations suggest that the convergence was driven by environmental changes during the Early and early Late Cretaceous, data on the environmental associations of these groups, particularly rebbachisaurids, are too scarce to confirm this. However, it is worth noting that of the 25 rebbachisaurids incorporated into this analysis, only one (the putative form, Amazonsaurus [Carvalho et al. 2003]) was recovered from a coastal environment, suggesting a possible "environmental convergence" between titanosaurs and rebbachisaurids.

Our data on the numbers of inland and coastal localities are based on localities where herbivorous dinosaurs are found. A more rigorous quantitative approach to analyzing environmental changes through the Jurassic and Cretaceous would include data on the areal extent of inland and coastal facies, including sediments that do not contain dinosaur fossils. A second line of inquiry would be to examine how potential forage plants are associated with habitat type and whether these plants declined in diversity and/or abundance during the Cretaceous.

\section{Wider Implications-Methodological Issues}

Although this study focuses on sauropod paleoecology, our analytical protocols and results raise issues that are of much wider significance, especially with regard to the methodology of establishing environmental associations. Some key points are discussed briefly below.

Body Fossils Compared with Trackways.Body fossils provide the bulk of information on sauropods. For example, in our data set 706 and 190 localities yielded sauropod body fossils and trackways respectively (Supplementary Materials; Fig. 3). Likewise, the contributions of body fossils and trackways to
Butler and Barrett's (2008) data set on Cretaceous herbivorous dinosaurs were $92.5 \%$ and $7.5 \%$ respectively. Body fossils also have the advantage of being assignable (often) to distinct clades (e.g., Brachiosauridae, Saltasauridae, Dicraeosauridae) or particular genera and species. However, body fossils can be transported to different environments after the animal dies, whereas trackways provide a direct record of where the animal actually stood while alive (Thulborn 1982; Lockley 1991; Wilson and Carrano 1999; Carrano and Wilson 2001). Our analyses provide an opportunity to compare the relative performances of trackway-based and body fossilbased data sets. A survey of Tables 2-10 reveals that both body fossil data (13 analyses) and trackway data (14 analyses) yield statistically significant results, and trackway data do not in general produce lower $p$-values than the body fossil data. If postmortem transport of body fossils has had a strong masking effect on habitat preferences, then the analyses based solely on trackways should have provided stronger support for environmental associations than those based just on body fossils.

Localities versus Individuals.-The use of numbers of individuals in this type of paleoecological study appears to be novel, at least with regard to vertebrates. Our definition of a "locality" is perhaps more arbitrary than the definition of an "individual," but because of the error that could be associated with estimating numbers of individuals from trackways or from fragmentary skeletons, we had expected that numbers of individuals would provide a less reliable guide to environmental associations. However, our analyses suggest that, if anything, individual-based analyses are more likely to find evidence for environmental associations than are locality-based ones. This phenomenon may be related to the fact that the number of individuals cannot be less than, and will often exceed, the number of localities. If the skew in the spatial distributions of the two taxon categories is subtle, then individualbased analyses will reveal the skew better because of the larger number of data points. Alternatively, even if there are substantial 
errors in the estimation of the numbers of individuals, these errors might be random with respect to environmental and taxon categories. If, for example, a habitat preference is expressed in terms of how much time each taxon spends in a given habitat, or the relative abundances of these taxa in each habitat, locality-based estimates of occurrences cannot capture this information because they record only presence or absence of a taxon. Thus, individual counts may be useful in capturing aspects of paleoecology that are ignored by locality-based counts, even when estimating the numbers of individuals is prone to significant error. This has parallels with modern ecological studies attempting to assess population size, where total counts are often impractical as a consequence of time, costs, and size of area (Waite 2000). Consequently, population size must be estimated with alternative techniques such as sample counts or capture-mark-recapture methods (Burnham and Overton 1979; Blower et al. 1981; Chao 1987; Waite 2000), using a variety of statistical approaches (see Colwell and Coddington 1994; Krebs 1999; Waite 2000; Sutherland 2006). Although some of these analytical methods have been implemented in paleoecological analyses (e.g., Harrington and Jaramillo 2007), they have yet to be applied to fossil vertebrates.

The Costs and Benefits of Time-Slicing and Sensitivity Analyses.-We have outlined above a hypothetical scenario in which parallel trends in the diversity of two taxon assemblages and the preservation rates of two environmental categories could create artifactual support for environmental associations. Exploring various subsets of the data can ameliorate these difficulties. One of the most useful approaches is to time slice the data in order to disrupt long-term trends in diversity and environmental preservation. Although such studies can reveal that putative environmental associations have changed through time, the key disadvantage of time-slicing is that as time slices become narrower, they include fewer data points. Thus, even though the data set as a whole contains such signals, numerous analyses may fail to find any statistically significant results (see Tables 3-9 for examples). Time-slicing is a key tool in the search for environmental associations because it allows the researcher to "fine-tune" the temporal range and taxonomic level of the proposed signals, but it should be applied with caution because it can be misleading about the taxonomic level at which a proposed environmental association occurs (e.g., Cretaceous "Macronaria" has virtually the same taxonomic content as "Titanosauriformes').

In this study, we re-analyzed our data after removing the Korean and U.S. tracksite data and found that our conclusions regarding environmental associations are affected by the presence or absence of these data blocks, although several analyses continue to support a positive association between titanosaurs and inland habitats (Table 10). Uneven sampling of the fossil record is a major topic of concern, particularly with regard to the temporal distributions of fossil taxa and the reconstruction of diversity curves (Raup 1972; Smith 2001; Peters and Foote 2001, 2002; Peters 2005, 2008; Upchurch and Barrett 2005; Smith and McGowan 2007; McGowan and Smith 2008; Barrett et al. 2009; Butler et al. 2009). The effect of uneven sampling on analyses of the spatial distribution of taxa has received considerably less attention, even though the ambiguity of "absence" likely affects both paleobiogeographic and paleoecological analyses (Ronquist 1997; Lieberman 2000; Hunn and Upchurch 2001; Upchurch and Hunn 2002). Ultimately, the analysis of the spatial distributions of fossil taxa might benefit from some form of rarefaction approach in which repeated subsamples of the data are selected at random and analyzed for associations or nonrandom area relationships. However, integration of such methods into the protocol for searching for environmental associations would require the creation of specialized software and lies outside of the scope of our study. Pending emergence of such software, we urge other researchers to explore their data sets by removing major blocks of data, changing the boundaries of time slices, and altering the criteria used to define localities and estimate numbers of individuals. 


\section{Conclusion}

Our results suggest that titanosaurs and non-titanosaurs display statistically significant associations with inland and coastal environments respectively. These signals occur when body fossils and trackway data are treated separately and together, for both locality-based and individual-based counts of occurrences. We interpret this pattern to mean that sauropod groups displayed habitat preferences, although the precise nature and strength of this preference are not clear at present. Wilson and Carrano's (1999) hypothesis that wide-gauge trackways were made by titanosaurs is reinforced by the observation that separate analyses of titanosaur body fossils and wide-gauge trackways display the same positive association with inland habitats. Finally, although the decline of non-titanosaurs and diversification of titanosaurs during the Early and early Late Cretaceous cannot be linked directly to habitat preferences, a better understanding of such preferences may help explain these events in the future.

Ecologists and invertebrate paleontologists have been investigating environmental associations for several decades, but the search for statistically robust associations among fossil vertebrate taxa is in its infancy. Although this lag is partly the result of suitable databases only recently becoming available (e.g., the Paleobiology Database), this is a long overdue focus for the field of vertebrate paleontology. It is crucial that paleobiologists test their ecological and evolutionary hypotheses using analytical methods and statistical tests that can distinguish genuine signals from the background noise generated by missing data and sampling biases. At the same time, these techniques must be applied, and their results interpreted, with subtlety and caution. The current study has demonstrated that both "total evidence" and time-slicing approaches have their costs and benefits, and that parallel trends in diversity and the representation of environments can create skews in spatial distributions that result in statistically significant but nonetheless artifactual support for environmental associations. We hope, therefore, that this study not only sheds some light on the evolution of sauropod dinosaurs, but also will stimulate more detailed quantitative analyses of ecological relationships in other extinct organisms.

\section{Acknowledgments}

We are grateful to the numerous institutions that allowed the study of material used in compiling the data set. The authors would like to express their gratitude to R. J. Butler for help with the statistical element of this work, as well as for providing data that was unpublished at the time, and to P. M. Barrett for helpful comments and discussion. Reviews by J. A. Wilson, M. Uhen, and C. Redman greatly improved the quality of this work, as did editorial comments by M. T. Carrano. Lastly, suggestions by N. Atkins greatly improved the clarity of this work. P. D. Mannion's research was supported by a University College London Natural Environment Research Council (NERC) studentship (NER/S/A/2006/14347).

\section{Literature Cited}

Aberhan, M., R. Bussert, W.-D. Heinrich, E. Schrank, S. Schultka, B. Sames, J. Kriwet, and S. Kapilima. 2002. Paleoecology and depositional environments of the Tendaguru Beds (Late Jurassic to Early Cretaceous, Tanzania). Mitteilungen aus dem Museum für Naturkunde in Berlin, Geowissenschaftliche Reihe 5:17-42.

Apesteguía, S. 2005. Evolution of the hyposphene-hypantrum complex within Sauropoda. Pp. 248-267 in Carpenter and Tidwell 2005.

Badgley, C. E. 1986. Counting individuals in mammalian fossil assemblages from fluvial environments. Palaios 1:328-338.

Barrett, P. M., and P. Upchurch. 2005. Sauropod diversity through time: possible macroevolutionary and paleoecological implications. Pp. 125-156 in Curry Rogers and Wilson 2005.

Barrett, P. M., A. J. McGowan, and V. Page. 2009. Dinosaur diversity and the rock record. Proceedings of the Royal Society B 276:2667-2674.

Blower, J. G., L. M. Cook, and J. A. Bishop. 1981. Estimating the size of animal populations. Allen and Unwin, London.

Bonaparte, J. F., W.-D. Heinrich, and R. Wild. 2000. Review of Janenschia Wild, with the description of a new sauropod from the Tendaguru beds of Tanzania and a discussion on the systematic value of procoelous caudal vertebrae in the Sauropoda. Palaeontographica, Abteilung A 256:25-76.

Borsuk-Bialynicka, M. 1977. A new camarasaurid sauropod Opisthocoelicaudia skarzynskii, gen. n., sp. n. from the Upper Cretaceous of Mongolia. Paleontologica Polonica 37:1-64.

Buffetaut, E., V. Suteethorn, G. Cuny, H. Tong, J. Le Loeuff, S. Khansubha, and S. Jongautchariyakul. 2000. The earliest known sauropod dinosaur. Nature 407:72-74.

Burnham, K. P., and W. S. Overton. 1979. Robust estimation of population size when capture probabilities vary among animals. Ecology 60:927-936. 
Butler, R. J., and P. M. Barrett. 2008. Paleoenvironmental controls on the distribution of Cretaceous herbivorous dinosaurs. Naturwissenschaften 95:1027-1032.

Butler, R. J., P. M. Barrett, P. Kenrick, and M. G. Penn. 2007. Paleoenvironmental controls on the distribution of Cretaceous herbivorous dinosaurs. Journal of Vertebrate Paleontology 27(Suppl. to No. 3):54A-55A.

Butler, R. J., P. M. Barrett, S. Nowbath, and P. Upchurch. 2009. Estimating the effects of the rock record on pterosaur diversity patterns: implications for hypotheses of bird/pterosaur competitive replacement. Paleobiology 35:432-446.

Calvo, J. O. 1994. Jaw mechanics in sauropod dinosaurs. In M. G. Lockley, V. F. dos Santos, C. A. Meyer, and A. P. Hunt, eds. Aspects of sauropod paleobiology. GAIA 10:183-193.

Carpenter, K., and V. Tidwell. 2005. Thunder lizards: the sauropodomorph dinosaurs. Indiana University Press, Bloomington.

Carrano, M. T. 2008. Taxonomy and classification of non-avian Dinosauria. Paleobiology Database Online Systematics Archive 4 (www.paleodb.org).

Carrano, M. T., and J. A. Wilson. 2001. Taxon distributions and the vertebrate track record. Paleobiology 27:563-581.

Carvalho, I. S., L. S. Avilla, and L. Salgado. 2003. Amazonsaurus maranhensis gen. et sp. nov. (Sauropoda, Diplodocoidea) from the Lower Cretaceous (Aptian-Albian) of Brazil. Cretaceous Research 24:697-713.

Chao, A. 1987. Estimating the population size for capturerecapture data with unequal capturability. Biometrics 43:783791.

Colwell, R. K., and J. A. Coddington. 1994. Estimating terrestrial biodiversity through extrapolation. Philosophical Transactions of the Royal Society of London B 345:101-118.

Curry Rogers, K. A. 2005. Titanosauria: a phylogenetic overview. Pp. 50-103 in Curry Rogers and Wilson 2005.

Curry Rogers, K. A., and C. A. Forster. 2001. The last of the dinosaur titans: a new sauropod from Madagascar. Nature 412:530-534.

- 2004. The skull of Rapetosaurus krausei (Sauropoda: Titanosauria) from the Late Cretaceous of Madagascar. Journal of Vertebrate Paleontology 24:121-144.

Curry Rogers, K. A., and J. A. Wilson. 2005. The sauropods: evolution and paleobiology. University of California Press, Berkeley.

Davis, E. B., and N. D. Pyenson. 2007. Diversity biases in terrestrial mammalian assemblages and quantifying the differences between museum collections and published accounts: a case study from the Miocene of Nevada. Palaeogeography, Palaeoclimatology, Palaeoecology 250:139-149.

Day, J. J., P. Upchurch, D. B. Norman, A. S. Gale, and H. P. Powell. 2002. Sauropod trackways, evolution, and behavior. Science 296:1659.

Day, J. J., D. B. Norman, A. S. Gale, P. Upchurch, and H. P. Powell. 2004. A Middle Jurassic dinosaur trackway site from Oxfordshire, UK. Palaeontology 47:319-348.

De Francesco, C. G., and G. S. Hassan. 2008. Dominance of reworked fossil shells in modern estuarine environments: implications for paleoenvironmental reconstructions based on biological remains. Palaios 23:14-23.

Dodson, P., A. K. Behrensmeyer, R. T. Bakker, and J. S. McIntosh. 1980. Taphonomy and paleoecology of the dinosaur beds of the Jurassic Morrison Formation. Paleobiology 6:208-232.

Farlow, J. O., J. G. Pittman, and J. M. Hawthorne. 1989. Brontopodus birdi, Lower Cretaceous sauropod footprints from the U.S. Gulf Coastal Plain. Pp. 371-394 in D. D. Gillette and M. G. Lockley, eds. Dinosaur tracks and traces. Cambridge University Press, Cambridge.

Gilinsky, N. L., and J. B. Bennington. 1994. Estimating numbers of whole individuals from collections of body parts: a taphonomic limitation of the paleontological record. Paleobiology 20:245258.

Gradstein, F. M., J. G. Ogg, and A. G. Smith. 2004. A geological timescale 2004. Cambridge University Press, Cambridge.

Grayson, D. K. 1973. On the methodology of faunal analysis. American Antiquity 39:432-439.

Hammer, Ø., and D. A. T. Harper. 2006. Paleontological data analysis. Blackwell, Oxford.

Harrington, G. J., and C. A. Jaramillo. 2007. Paratropical floral extinction in the Late Paleocene-Early Eocene. Journal of the Geological Society, London 164:323-332.

Henderson, D. M. 2006. Burly gaits: centers of mass, stability, and the trackways of sauropod dinosaurs. Journal of Vertebrate Paleontology 26:907-921.

Hunn, C. A., and P. Upchurch. 2001. The importance of time/ space in diagnosing the causality of phylogenetic events: towards a "chronobiogeographical" paradigm? Systematic Biology 50:1-17.

Hutchinson, J. R., V. Ng-Thow-Hing, and F. C. Anderson. 2007. A 3D interactive method for estimating body segmental parameters in animals: application to the turning and running performance of Tyrannosaurus rex. Journal of Theoretical Biology 246:660-680.

Jacobs, L. L., D. A. Winkler, W. R. Downs, and E. M. Gomani. 1993. New material of an Early Cretaceous titanosaurid sauropod dinosaur from Malawi. Paleontology 36:523-534.

Janensch, W. 1929. Material und Formegehalt der Sauropoden in der Ausbeute der Tendaguru-Expedition, 1909-1912. Palaeontographica 2(Suppl. VII):3-34.

Krebs, C. J. 1999. Ecological methodology, 2d ed. Addison-Wesley Longman, Menlo Park, Calif.

Lee, Y.-N., S.-Y. Yang, S.-J. Seo, K.-S. Baek, D.-J. Lee, E.-J. Park, and S.-W. Han. 2000. Distribution and paleobiological significance of dinosaur tracks in the Jindong Formation (Albian) in Kosong County, Korea. Paleontological Society of Korea Special Publication 4:1-12.

Lehman, T. M. 1987. Late Maastrichtian paleoenvironments and dinosaur biogeography in the western interior of North America. Palaeogeography, Palaeoclimatology, Palaeoecology 60:189-217.

Lieberman, B. S. 2000. Paleobiogeography: using fossils to study global change, plate tectonics, and evolution. Kluwer Academic /Plenum, New York.

Lim, S.-K., S.-Y. Yang, and M. G. Lockley. 1989. Large dinosaur footprint assemblages from the Cretaceous Jindong Formation of southern Korea. Pp. 333-336 in D. D. Gillette and M. G. Lockley, eds. Dinosaur tracks and traces. Cambridge University Press, Cambridge.

Lockley, M. G. 1991. Tracking dinosaurs: a new look at an ancient world. Cambridge University Press, Cambridge.

Lockley, M. G., C. A. Meyer, A. P. Hunt, and S. Lucas. 1994. The distribution of sauropod tracks and trackmakers. In M. G. Lockley, V. F. dos Santos, C. A. Meyer, and A. P. Hunt, eds. Aspects of sauropod paleobiology. GAIA 10:233-248.

Lockley, M. G., K. Houck, S.-Y. Yang, M. Matsukawa, and S.-K. Lim. 2006. Dinosaur-dominated footprint assemblages from the Cretaceous Jindong Formation, Hallayo Haesang National Park, Goseong County, South Korea: evidence and implications. Cretaceous Research 27:20-101.

Lucas, S. G., and A. P. Hunt. 1989. Alamosaurus and the sauropod hiatus in the Cretaceous of the North American western interior. In J. O. Farlow, ed. Paleobiology of the dinosaurs. Geological Society of America Special Paper 238:75-85.

McGowan, A. J., and A. B. Smith. 2008. Are global Phanerozoic marine diversity curves truly global? A study of the relationship between regional rock records and global Phanerozoic marine diversity. Paleobiology 34:80-103. 
McIntosh, J. S. 1990. Sauropoda. Pp. 345-401 in D. B. Weishampel, P. Dodson, and H. Osmólska, eds. The Dinosauria, 1st ed. University of California Press, Berkeley.

Milàn, J., and R. G. Bromley. 2006. True tracks, undertracks and eroded tracks, experimental work with tetrapod tracks in laboratory and field. Palaeogeography, Palaeoclimatology, Palaeoecology 231:253-264.

Ostrom, J. H., and J. S. McIntosh. 1966. Marsh's dinosaurs. Yale University Press, New Haven, Conn.

Peters, S. E. 2005. Geological constraints on the macroevolutionary history of marine animals. Proceedings of the National Academy of Sciences USA 102:12,326-12,331.

- 2008. Environmental determinants of extinction selectivity in the fossil record. Nature 454:626-629.

Peters, S. E., and K. B. Bork. 1999. Species-abundance models: an ecological approach to inferring paleoenvironment and resolving paleoecological change in the Waldron Shale (Silurian). Palaios 14:234-245.

Peters, S. E., and M. Foote. 2001. Biodiversity in the Phanerozoic: a reinterpretation. Paleobiology 27:583-601.

2002. Determinants of extinction in the fossil record. Nature 416:420-424.

Powell, J. E. 1992. Osteología de Saltasaurus loricatus (SauropodaTitanosauridae) del Cretácico Superior del Noroeste Argentino. Pp. 165-230 in J. L. Sanz and A. D. Buscalioni, eds. Los dinosaurios y su entorno biotico. Instituto "Juan de Valdes," Cuenca, Spain.

Raup, D. M. 1972. Taxonomic diversity during the Phanerozoic Science 177:1065-1071.

Rice, W. R. 1989. Analyzing tables of statistical tests. Evolution 43:223-225.

Ronquist, F. 1997. Dispersal-vicariance analysis: a new biogeographic approach to the quantification of historical biogeography. Systematic Biology 46:195-203.

Russell, D., P. Beland, and J. S. McIntosh. 1980. Paleoecology of the dinosaurs of Tendaguru (Tanzania). Mémoires de la Société Géologique de France 139:169-175.

Salgado, L. 2001. Los saurópodos de Patagonia: sistemátuca, evolucíon y paleobiología. Pp. 139-168 in Actas de Las II Journadas Internacionales sobre Paleontología de Dinosaurios y su Entorno. Salas de los Infantes, Burgos, Spain.

Salgado, L., R. A. Coria, and J. O. Calvo. 1997. Evolution of titanosaurid sauropods. I. Phylogenetic analysis based on the postcranial evidence. Ameghiniana 34:3-32.

Scholz, H., and J. H. Hartman. 2007. Paleoenvironmental reconstruction of the Upper Cretaceous Hell Creek Formation of the Williston Basin, Montana, USA: implications from the quantitative analysis of unionoid bivalve taxonomic diversity and morphologic disparity. Palaios 22:24-34.

Sepkoski, J. J., Jr. 1984. A kinematic model of Phanerozoic taxonomic diversity. III. Post-Paleozoic families and mass extinctions. Paleobiology 10:246-267.

Sereno, P. C. 1999. The evolution of dinosaurs. Science 284:21372147.

Smith, A. B. 2001. Large-scale heterogeneity of the fossil record: implications for Phanerozoic biodiversity studies. Philosophical Transactions of the Royal Society of London B 356:351-367.

Smith, A. B., and A. J. McGowan. 2007. The shape of the Phanerozoic marine paleodiversity curve: how much can be predicted from the sedimentary rock record of Western Europe? Paleontology 50:765-774.

Sokal, R. R., and F. J. Rohlf. 1987. Introduction to biostatistics. W. H. Freeman, New York.
Sutherland, W. J. 2006. Ecological census techniques: a handbook, 2d ed. Cambridge University Press, Cambridge.

Thulborn, R. A. 1982. Speeds and gaits of dinosaurs. Palaeogeography, Palaeoclimatology, Palaeoecology 38:227-256.

Upchurch, P. 1995. Evolutionary history of sauropod dinosaurs. Philosophical Transactions of the Royal Society of London B 349:365-390.

1999. The phylogenetic relationships of the Nemegtosauridae (Saurischia, Sauropoda). Journal of Vertebrate Paleontology 19:106-125.

Upchurch, P., and P. M. Barrett. 2000. The evolution of sauropod feeding mechanisms. Pp. 79-122 in H.-D. Sues, ed. The evolution of herbivory in terrestrial vertebrates: perspectives from the fossil record. Cambridge University Press, Cambridge. 2005. A phylogenetic perspective on sauropod diversity. Pp. 104-124 in Curry Rogers and Wilson 2005.

Upchurch, P., C. A. Hunn, and D. B. Norman. 2002. An analysis of dinosaurian biogeography: evidence for the existence of vicariance and dispersal patterns caused by geological events. Proceedings of the Royal Society of London B 269:613-622.

Upchurch, P., P. M. Barrett, and P. Dodson. 2004. Sauropoda. Pp. 259-322 in Weishampel et al. 2004b.

Waite, S. 2000. Statistical ecology in practice: a guide to analysing environmental and ecological field data. Pearson Education Limited, Harlow, U.K.

Weishampel, D. B., P. M. Barrett, R. E. Coria, J. Le Loeuff, E. S. Gomani, Z. Zhao, X. Xu, A. Sahni, and C. Noto. 2004a. Dinosaur distribution. Pp. 517-606 in Weishampel et al. 2004b.

Weishampel, D. B., P. Dodson, and H. Osmólska, eds. 2004b. The Dinosauria, 2d ed. University of California Press, Berkeley.

Wilson, J. A. 2002. Sauropod dinosaur phylogeny: critique and cladistic analysis. Zoological Journal of the Linnean Society 136:217-276.

- 2005a. Integrating ichnofossils and body fossil records to estimate locomotor posture and spatiotemporal distribution of early sauropod dinosaurs: a stratocladistic approach. Paleobiology 31:400-423.

- 2005b. A redescription of the skull of Nemegtosaurus mongoliensis (Dinosauria-Sauropoda) and its relevance to Cretaceous titanosaur diversity. Journal of Systematic Paleontology 3:283-318.

Wilson, J. A., and M. T. Carrano. 1999. Titanosaurs and the origin of "wide-gauge" trackways: a biomechanical and systematic perspective on sauropod locomotion. Paleobiology 25: 252-267.

Wilson, J. A., and P. C. Sereno. 1998. Early evolution and higherlevel phylogeny of sauropod dinosaurs. Society of Vertebrate Paleontology Memoir 5:1-68.

Wilson, J. A., and P. Upchurch. 2003. A revision of Titanosaurus (Dinosauria Sauropoda), the first "Gondwanan" dinosaur genus. Journal of Systematic Paleontology 1:125-60.

- 2009. Redescription and reassessment of the phylogenetic affinities of Euhelopus zdanskyi (Dinosauria: Sauropoda) from the Late Jurassic or Early Cretaceous of China. Journal of Systematic Paleontology 7:199-239.

Wright, J. L. 2005. Steps in understanding sauropod biology: the importance of sauropod tracks. Pp. 252-284 in Curry Rogers and Wilson 2005.

Yates, A. M., and J. W. Kitching. 2003. The earliest known sauropod dinosaur and the first steps towards sauropod locomotion. Proceedings of the Royal Society of London B 270:1753-1758. 